\title{
ALMA Observations Show Major Mergers Among the Host Galaxies of Fast- growing, High-redshift, Supermassive Black Holes
}

\author{
Benny Trakhtenbrot ${ }^{1,8}$, Paulina Lira ${ }^{2}$, Hagai Netzer ${ }^{3}$, Claudia Cicone ${ }^{1,4}$, Roberto Maiolino ${ }^{5,6}$, and Ohad Shemmer ${ }^{7}$ \\ ${ }^{1}$ Institute for Astronomy, Department of Physics, ETH Zurich, Wolfgang-Pauli-Strasse 27, CH-8093 Zurich, Switzerland; benny.trakhtenbrot@phys.ethz.ch \\ ${ }^{2}$ Departamento de Astronomia, Universidad de Chile, Camino del Observatorio 1515, Santiago, Chile \\ ${ }^{3}$ School of Physics and Astronomy and the Wise Observatory, The Raymond and Beverly Sackler Faculty of Exact Sciences, \\ Tel-Aviv University, Tel-Aviv 69978, Israel \\ ${ }^{4}$ INAF-Osservatorio Astronomico di Brera, via Brera 28, I-20121, Milano, Italy \\ ${ }^{5}$ Cavendish Laboratory, University of Cambridge, 19 J. J. Thomson Avenue, Cambridge CB3 0HE, UK \\ ${ }^{6}$ Kavli Institute for Cosmology, University of Cambridge, Madingley Road, Cambridge CB3 OHA, UK \\ 7 Department of Physics, University of North Texas, Denton, TX 76203, USA \\ Received 2016 August 22; revised 2016 November 25; accepted 2016 December 9; published 2017 February 3
}

\begin{abstract}
We present new ALMA band-7 data for a sample of six luminous quasars at $z \simeq 4.8$, powered by fast-growing supermassive black holes (SMBHs) with rather uniform properties: the typical accretion rates and black hole masses are $L / L_{\mathrm{Edd}} \simeq 0.7$ and $M_{\mathrm{BH}} \simeq 10^{9} M_{\odot}$. Our sample consists of three "FIR-bright" sources, which were individually detected in previous Herschel/SPIRE observations, with star formation rates of SFR $>1000 M_{\odot} \mathrm{yr}^{-1}$, and three "FIR-faint" sources for which Herschel stacking analysis implies a typical SFR of $\sim 400 M_{\odot} \mathrm{yr}^{-1}$. The dusty interstellar medium in the hosts of all six quasars is clearly detected in the ALMA data and resolved on scales of $\sim 2 \mathrm{kpc}$, in both continuum $\left(\lambda_{\text {rest }} \sim 150 \mu \mathrm{m}\right)$ and [C II] $\lambda 157.74 \mu \mathrm{m}$ line emission. The continuum emission is in good agreement with the expectations from the Herschel data, confirming the intense SF activity in the quasar hosts. Importantly, we detect companion sub-millimeter galaxies (SMGs) for three sources-one FIR-bright and two FIR-faint, separated by $\sim 14-45 \mathrm{kpc}$ and $<450 \mathrm{~km} \mathrm{~s}^{-1}$ from the quasar hosts. The [C II]-based dynamical mass estimates for the interacting SMGs are within a factor of $\sim 3$ of the quasar hosts' masses, while the continuum emission implies $\mathrm{SFR}_{\text {quasar }} \sim(2-11) \times \mathrm{SFR}_{\mathrm{SMG}}$. Our ALMA data therefore clearly support the idea that major mergers are important drivers for rapid early SMBH growth. However, the fact that not all high-SFR quasar hosts are accompanied by interacting SMGs and the gas kinematics as observed by ALMA suggest that other processes may be fueling these systems. Our analysis thus demonstrates the diversity of host galaxy properties and gas accretion mechanisms associated with early and rapid SMBH growth.
\end{abstract}

Key words: galaxies: active - galaxies: high-redshift - galaxies: interactions - galaxies: star formation - quasars: general

\section{Introduction}

Several lines of evidence suggest that the growth histories of Supermassive Black Holes (SMBHs) are closely linked to that of their host galaxies. These include the well-known scaling relations between the SMBH mass $\left(M_{\mathrm{BH}}\right)$ and several properties of the (bulge component of the) hosts, observed in local relic systems (see Kormendy \& Ho 2013 for a recent review); and the coincidence of intense star formation (SF) and SMBH growth, at higher redshifts. Indeed, for systems dominated by accretion onto the SMBH-identified as active galactic nuclei (AGNs) - the luminosities associated with SMBH accretion $\left(L_{\mathrm{AGN}}\right)$ and with star formation $\left(L_{\mathrm{SF}}\right)$ are correlated over several orders of magnitude (e.g., Netzer 2009; Lutz et al. 2010; Shao et al. 2010; Rosario et al. 2013, but see also Page et al. 2012). This suggests that the phase of fast SMBH growth occurs in tandem with intense SF activity, reaching star formation rates (SFRs) of $\sim 1000 M_{\odot} \mathrm{yr}^{-1}$ for SMBHs with $\dot{M}_{\mathrm{BH}} \sim 15 M_{\odot} \mathrm{yr}^{-1}$ (i.e., $L_{\mathrm{AGN}} \sim 10^{47} \mathrm{erg} \mathrm{s}^{-1}$ ). All this supports a general idea that both processes (SF and AGNs) are fed by a common reservoir of cold gas that collapses, forms stars, and (eventually) reaches the central region of the host galaxy to be accreted by the SMBH.

\footnotetext{
${ }^{8}$ Zwicky Postdoctoral Fellow.
}

A particularly popular framework for the co-evolution of SMBHs and their hosts focuses on major mergers between massive, gas-rich galaxies. Theoretical studies highlight the ability of such mergers to account for both the observed properties of AGN and SF galaxies, and for the SMBH-host relations in relic systems (see, e.g., Sanders et al. 1988; Di Matteo et al. 2005; Hopkins et al. 2006 and the review by Alexander \& Hickox 2012). In particular, many simulations of such mergers show a relatively short episode (on the order of $100 \mathrm{Myr}$ ) of parallel intense SF and AGN activity, with SFRs reaching several hundred $M_{\odot} \mathrm{yr}^{-1}$ (or exceeding $\sim 1000 M_{\odot} \mathrm{yr}^{-1}$ in some simulations; see, e.g., Blecha et al. 2011; DeBuhr et al. 2011; Sijacki et al. 2011; Capelo et al. 2015; Volonteri et al. 2015; Gabor et al. 2016). Observationally, however, the relevance of mergers to fast SMBH growth, and indeed to the co-evolutionary framework, is not yet well established. While some studies of low$z$ luminous AGNs have reported a high occurrence of mergers (e.g., Bahcall et al. 1997; Veilleux et al. 2009; Koss et al. 2011), several other studies have demonstrated that interacting AGN hosts are relatively rare at $1 \lesssim z \lesssim 2$ and their occurrence rate does not exceed what was found for non-active galaxies (e.g., Gabor et al. 2009; Bennert et al. 2011; Cisternas et al. 2011; Mainieri et al. 2011; Schawinski et al. 2011, 2012; Kocevski et al. 2012). A possible explanation for these apparently contradictory results, as put forward by Treister 
et al. (2012), is that the merger-driven scenario may only be relevant for the epochs of fastest growth of the most massive $\mathrm{BH}$ (that is, highest $L_{\mathrm{AGN}}$ ), and at $z>2$-when the overall rate of major mergers is expected to be higher (e.g., Genel et al. 2009; Hopkins et al. 2010) and the amount of gas in the relevant halos is considerably larger (but see also Dubois et al. 2015).

As the relevance of mergers to $\mathrm{SMBH}$ growth is still debated, several recent studies have highlighted the importance of alternatives to the merger scenario. Direct flows of cold gas from the intergalactic medium (IGM; e.g., Dekel et al. 2009; Di Matteo et al. 2012; Dubois et al. 2012), which may also trigger "secular" instabilities of the gas or the stars in the close environment of the SMBH (e.g., Springel et al. 2005; Bournaud et al. 2011, 2012), are claimed to be most relevant for the early and fast growth of high-redshift SMBHs. Such models, however, usually produce SFRs of only a few hundred $M_{\odot} \mathrm{yr}^{-1}$. All this suggests that the best way to test and understand the relevance of the merger-driven scenario for SMBH growth is to focus on well-defined samples of fastgrowing SMBHs, preferably at early cosmic epochs $(z>4)$, when the most massive BHs were growing at maximal rates (Trakhtenbrot et al. 2011; Trakhtenbrot \& Netzer 2012; De Rosa et al. 2014). Another consequence of the aforementioned scenarios is that such fast-growing SMBHs would be predominantly found in significantly over-dense large-scale environments, where the rate of mergers is yet higher and where cold gas streams are expected to converge and provide ample gas supply to the SMBHs (e.g., Di Matteo et al. 2008; Sijacki et al. 2009; Costa et al. 2014).

Testing these ideas observationally is, however, extremely challenging. The AGN-related emission dominates over most of the optical-NIR spectral regime, significantly limiting the prospects of determining the host properties. The cosmic environments of the SMBHs are often characterized by searching for nearby (rest-frame) UV-bright galaxies, without precise redshift determinations, and possibly missing dusty obscured SF galaxies. Indeed, several studies provided ambiguous evidence for over-densities around some, but definitely not all $z \gtrsim 5$ quasars (e.g., Willott et al. 2005; Overzier et al. 2006; Kim et al. 2009; Utsumi et al. 2010; Bañados et al. 2013; Husband et al. 2013; Simpson et al. 2014, and references therein).

The advent of large and sensitive sub-millimeter (sub-mm) interferometric arrays, such as the IRAM Plateau de Bure interferometer (now NOEMA) and the Atacama Large Millimeter/sub-millimeter Array (ALMA), has enabled the direct observation of the hosts of high-redshift quasars in a spectral regime that is mostly uncontaminated by the AGN emission. The early study of Iono et al. (2006, using the SMA) demonstrated the ability of such data to reveal major mergers among quasar hosts, presenting a close merger between two $z=4.7 \mathrm{SF}$ galaxies powering a pair of AGNs, one of which is a luminous quasar. The ALMA study of the same system (Wagg et al. 2012) showcased the revolutionary increase of spatial resolution and sensitivity provided by ALMA. In recent years, a growing number of $z \gtrsim 5$ quasars were studied with various sub-mm facilities (e.g., Maiolino et al. 2005; Walter et al. 2009; Gallerani et al. 2012; Venemans et al. 2012, 2016; Wang et al. 2013; Willott et al. 2013, 2015; Cicone et al. 2015), focusing on the continuum emission, which originates from SFheated dust in the interstellar medium (ISM) of the hosts, and the [C II] $\lambda 157.74 \mu \mathrm{m}$ emission line, which is among the most efficient ISM coolants (e.g., Stacey et al. 1991, 2010; Carilli \& Walter 2013, and references therein). These studies have provided additional support for the coexistence of fast-growing SMBHs and intense SF activity in their hosts, but mostly could not address the questions related to the merger-driven scenario, due to limited sensitivity, resolution, and/or field of view. Indeed, no major mergers were identified in the aforementioned studies of high-redshift AGNs.

In this study, we focus on a sample of quasar hosts at $z \simeq 4.8$, for which we have accumulated a wealth of multiwavelength data. In our previous studies, we have shown that the SMBHs powering these quasars trace an epoch of fast Eddington-limited growth from massive $\mathrm{BH}$ seeds, which is expected to form the most massive BHs known by $z \sim 4$ (Trakhtenbrot et al. 2011, T11 hereafter). Our Herschel/SPIRE campaign (Mor et al. 2012; Netzer et al. 2014, M12 and N14 hereafter) showed that these fast-growing SMBHs are hosted by $\mathrm{SF}$ galaxies, with $\sim 1 / 4$ of the systems exceeding SFR $\sim 1000 M_{\odot} \mathrm{yr}^{-1}$, while a stacking analysis of the other $\sim 3 / 4$ of the systems suggested a typical SFR of $\sim 400 M_{\odot} \mathrm{yr}^{-1}$ (see also Netzer et al. 2016). The extremely high SFRs found for the FIR-bright sources were interpreted as tracing major merger activity, while the lower SFRs found for most systems were thought to be tracing the early stages of SF suppression by AGN-driven "feedback." The poor spatial resolution of the Herschel data $\left(\sim 18^{\prime \prime}\right.$, or $\left.\gtrsim 100 \mathrm{kpc}\right)$ was, however, insufficient to test these ideas, which can be now addressed directly with the ALMA FIR continuum and [C II] $\lambda 157.74 \mu \mathrm{m}$ emission line observations presented in this study. In Section 2, we describe the sample, the ALMA observations, data reduction, and analysis. In Section 3, we compare the ISM properties and the occurrence of close (interacting) companions to those found in other samples of high-redshift AGNs. We summarize our main findings in Section 4. Throughout this work, we assume a cosmological model with $\Omega_{\Lambda}=0.7, \Omega_{\mathrm{M}}=0.3$, and $H_{0}=70 \mathrm{~km} \mathrm{~s}^{-1} \mathrm{Mpc}^{-1}$, which provides an angular scale of about $6.47 \mathrm{kpc} /{ }^{\prime \prime}$ at $z=4.7$-the typical redshift of our sources. We further assume the stellar initial mass function (IMF) of Chabrier (2003).

\section{Sample, ALMA Observations, and Data Analysis}

\subsection{Sample Selection and Properties}

Our targets are drawn from a flux-limited sample of 40 luminous, unobscured quasars at $z \simeq 4.8$, which we have studied in detail in a series of previous publications. Here, we only briefly mention the sample selection and the ancillary data available for our targets and we refer the reader to our previous papers (T11, M12, N14) for additional details. This $z \simeq 4.8$ quasar sample was originally selected from the sixth data release of the Sloan Digital Sky Survey (SDSS/DR6; York et al. 2000; Adelman-McCarthy et al. 2008). The sample spans a narrow redshift range of $z \sim 4.65-4.92$, to enable follow-up near-IR spectroscopy of the broad Mg II $\lambda 2798$ emission line. Such near-IR spectroscopy was indeed performed using the VLT/SINFONI and Gemini-North/NIRI instruments and has provided reliable estimates of the masses $\left(M_{\mathrm{BH}}\right)$ and normalized 
accretion rates $\left(L / L_{\mathrm{Edd}}\right)$ of the quasars (T11). These clearly showed that the $z \simeq 4.8$ quasars are powered by fast-growing SMBHs with typical masses of $M_{\mathrm{BH}} \simeq 8 \times 10^{8} M_{\odot}$ and accretion rates of $L / L_{\mathrm{Edd}} \simeq 0.6$ (median values), representing the epoch of fastest growth for the most massive BHs.

A follow-up Herschel/SPIRE campaign targeted these quasars, probing the peak of the SF-heated dust continuum emission (M12, N14). These data provided robust continuum detections for $\sim 1 / 4$ of the quasars, with FIR luminosities of $L_{\mathrm{FIR}} \sim 2.4 \times 10^{13} L_{\odot}, \quad$ suggesting SFRs in the range $\sim 1000-4000 M_{\odot} \mathrm{yr}^{-1}$. A stacking analysis of the remaining $\sim 3 / 4$ of the quasars revealed a median SFR of $\sim 400 M_{\odot} \mathrm{yr}^{-1}$ (see the refined stacking analysis in Netzer et al. 2016). Importantly, these "FIR-bright" and "FIR-faint" sources are highly uniform in terms of the SMBH-related properties that drive the AGN emission (i.e., $L_{\mathrm{AGN}}, M_{\mathrm{BH}}$, and hence $L / L_{\mathrm{Edd}}$ ), with a tendency of the FIR-bright sources to have somewhat higher $M_{\mathrm{BH}}$ and $L_{\mathrm{AGN}}$ (by $\lesssim 0.4 \mathrm{dex}$ ). Thus, the Herschel data presented in M12 and N14 suggests a wide variety of SF activity among an almost uniform sample of fast-growing SMBHs in the early universe, with SFRs that range over an order of magnitude, compared with a significantly narrower range of basic SMBH properties. The N14 study also presented the results of a dedicated Spitzer/IRAC campaign targeting almost all of the T11 quasars, at observed-frame 3.6 and 4.5 $\mu \mathrm{m}$. These data were used to derive positional priors that allowed us to de-blend the low-resolution Herschel/ SPIRE data.

A comparison of the FIR luminosities of the quasar hosts (implied from the Herschel data) to the AGN-related luminosities (i.e., the bolometric luminosities from T11), implies that the FIR-bright sources are so FIR-luminous, that they reach $L_{\mathrm{SF}} \simeq L_{\mathrm{AGN}}$. The FIR-faint systems, on the other hand, have $L_{\mathrm{AGN}} \gtrsim 5 \times L_{\mathrm{SF}}$, making them "AGN dominated."

The current study focuses on six objects selected from our parent T11 quasar sample, split equally between three FIRbright and three FIR-faint objects. We chose to focus on the lower-redshift sources among our parent sample $(z<4.8)$ to avoid the $[\mathrm{C} \mathrm{II}]$ emission line to be redshifted into the lowatmospheric transmission region near $325 \mathrm{GHz}$. The redshifts of the selected targets are in the range $z=4.658-4.729$, with a median of $z_{\text {median }}=4.6703$ (as determined from the $\mathrm{Mg}$ II lines; see Section 3.2 for more details). We will nonetheless refer to them here as " $z \simeq 4.8$ quasars," following our previous studies. The targets are all equatorial, so to allow efficient ALMA observations. In practice, this implies that the NIR data for all the six quasars studied here were obtained with the VLT/SINFONI. These redshift and declination restrictions leave only 4 of the 10 Herschel-detected FIR-bright quasars reported in N14, of which we finally chose 3 . As for the 3 FIRfaint targets, we focused on those with higher quality $\mathrm{Mg}$ II fits, as assessed in T11. ${ }^{9}$ We stress that all these selection criteria do not introduce any biases, in terms of the a-priori known SMBH- and host-related properties of the systems under study. In particular, the $M_{\mathrm{BH}}$ values of the two sub-groups are consistent, within the errors (except for the relatively high-mass object J1341; see Table 4).

\footnotetext{
9 In terms of both flux calibration and spectral fit quality; corresponding to " $L$-quality" and "FWHM(Mg II)-quality" flags of 1 or 2 in Table 2 of T11.
}

\subsection{Observations, Data Reduction, and Analysis}

\subsubsection{ALMA Observations}

The six targets were observed in ALMA band-7, as part of cycle-2 (project code 2013.1.01153.S), during the period 2014 July 18 to 2015 June 13 . As the capabilities of ALMA were expanded during this period, the data presented here were collected with a varying number of $12 \mathrm{~m}$ antennas, between 2938. The observations were set up to use the extended C34-4 configuration, which provides a resolution of about 0.3 at $330 \mathrm{GHz}$ (corresponding to about $2 \mathrm{kpc}$ at $z \simeq 4.8$ ). We aimed at spectrally resolving the [C II] emission line, which is expected to have a width of several hundred $\mathrm{km} \mathrm{s}^{-1}$ and also to cover a wide spectral range that includes line-free continuum regions.

We chose to use the TDM correlator mode, providing four spectral windows, each covering an effective bandwidth of $1875 \mathrm{MHz}$, corresponding to $\sim 1650 \mathrm{~km} \mathrm{~s}^{-1}$ at the observed frequencies. This spectral range is sampled by 128 channels, providing a spectral resolution of $\sim 30 \mathrm{~km} \mathrm{~s}^{-1}$. One such spectral window was centered on the frequency corresponding to the expected peak of the [C II] line, given the $\mathrm{Mg}$ II-based redshifts of our targets (as determined in T11). The other three bands extended to higher frequencies, with the first being adjacent to the $[\mathrm{C} \mathrm{II}]$-centered band and the other two separated from this first pair by about $12 \mathrm{GHz}$. Each of these two pairs of bands included a small overlapping spectral region, of roughly $50 \mathrm{MHz}$. In addition, the rejection of a few of the channels at the edge of the spectral bands, due to divergent flux values (a common flagging procedure in ALMA data reduction), implies that in some cases a small gap is seen between windows. Given this spectral setup of four bands, the ALMA observations could in principle probe [C II] line emission over a spectral region with a width corresponding to roughly $\Delta z \simeq 0.06$.

Additional details regarding the ALMA observations, including the full object names of our sources, are given in Table 1. For clarity, we use abbreviated object names (i.e., "JHHDD") throughout the rest of the paper.

The ALMA band-7 flux densities reach a depth of $F_{\nu} \simeq(4.2-9.2) \times 10^{-2} \mathrm{mJy} /$ beam (rms). This flux limit can be translated to a limit on our ability to detect dusty, SF galaxies, at the redshift range of interest. If we assume the same gray-body FIR spectral energy distribution (SED) as we do in our analysis of the quasars hosts' - a dust temperature of $T_{\mathrm{d}}=47 \mathrm{~K}$ and a power-law exponent of $\beta=1.6$, and the scaling between FIR luminosity and SFR appropriate for our chosen IMF (see Section 3.3), this corresponds to $3 \sigma$ upper limits on the SFR in the range $\sim 20-50 M_{\odot} \mathrm{yr}^{-1}$, per beam. Given the beam sizes of our ALMA observations (Table 1), these translate to $\sim 4-11 M_{\odot} \mathrm{yr}^{-1} / \mathrm{kpc}^{2}$.

\subsubsection{Data Reduction and Spectral Measurements}

Data reduction was performed using CASA package, version 4.5.0 (Mcmullin et al. 2007). The scripts provided by the observatory were used to generate the visibilities. We then applied the CLEAN algorithm, using a "natural" weighting to determine the noise level for each observation by averaging over the three line-free spectral windows. The resulting flux density sensitivities and synthesized beam sizes are presented in Table 1. We note that self calibration for the brightest sources did not improve the signal-to-noise ratios and therefore was not implemented. 
Table 1

Observations Log

\begin{tabular}{|c|c|c|c|c|c|c|c|}
\hline sub-sample & $\begin{array}{l}\text { Target } \\
\text { ID }\end{array}$ & $N_{\text {Ant }}{ }^{\mathrm{a}}$ & $\begin{array}{c}T_{\exp } \\
\mathrm{S}\end{array}$ & $\begin{array}{c}F_{\nu} \mathrm{rms}^{\mathrm{b}} \\
\mathrm{mJy} / \text { beam }\end{array}$ & $\begin{array}{c}\text { Beam Size } \\
\square^{\prime \prime}\end{array}$ & $\begin{array}{c}\text { Pixel Size } \\
\end{array}$ & ALMA Companions \\
\hline \multirow[t]{3}{*}{ Bright } & SDSS J033119.67-074143.1 & 29 & 792 & $9.2 \times 10^{-2}$ & $0.41 \times 0.31$ & 0.06 & $\ldots$ \\
\hline & SDSS J134134.20+014157.7 & 35 & 697 & $5.6 \times 10^{-2}$ & $0.38 \times 0.30$ & 0.06 & $\ldots$ \\
\hline & SDSS J151155.98+040803.0 & 30 & 729 & $8.7 \times 10^{-2}$ & $0.53 \times 0.31$ & 0.06 & $1 \mathrm{SMG}(\mathrm{w} /[\mathrm{C}$ II $])$ and 1 "blob" (w/o [C II] $)$ \\
\hline \multirow[t]{3}{*}{ Faint } & SDSS J092303.53+024739.5 & 38 & 2978 & $4.3 \times 10^{-2}$ & $0.51 \times 0.29$ & 0.06 & $1 \mathrm{SMG}(\mathrm{w} /[\mathrm{C}$ II $])$ \\
\hline & SDSS J132853.66-022441.6 & 36 & 2852 & $4.2 \times 10^{-2}$ & $0.48 \times 0.31$ & 0.06 & $1 \mathrm{SMG}(\mathrm{w} /[\mathrm{C}$ II $])$ \\
\hline & SDSS J093508.49+080114.5 & $35-33$ & 3230 & $5.1 \times 10^{-2}$ & $0.54 \times 0.29$ & 0.06 & 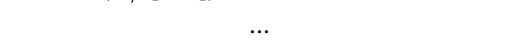 \\
\hline
\end{tabular}

Notes.

${ }^{a}$ Number of antennae used, averaging after antennae flagging.

b Flux densities, averaging over the three continuum (line-free) spectral windows. CLEAN performed with weighting = "natural."

Both continuum and emission line images were created by applying the CLEAN algorithm using a "briggs" weighting (with robustness parameter set to 0.5), to obtain images with the best possible spatial resolution. Continuum emission images were constructed using the two highest frequency, line-free spectral windows. [C II] emission line images were constructed by subtracting the continuum emission from the other two, lower frequency spectral windows, in the UV space (using standard CASA procedures). We verified that the resulting [C II] emission line images have no residual continuum signal in them. The sizes of the continuum- and line-emitting regions were determined from the respective images by fitting two-dimensional Gaussians. These sizes are given in Table 2. Velocity and velocity dispersion maps (i.e., second- and third-moment maps) were obtained from the line images using the standard CASA procedure.

The properties of the [C II] emission lines were measured from the two lower frequency spectral windows of the continuum-subtracted "briggs" weighted cubes. The integrated line fluxes were measured through two different procedures. In the first ("spatial") approach, we created zero-moment images (i.e., integrated over the spectral axes) for all sources and fitted the spatial distribution of line emission with 2D Gaussian profiles, which are characterized by a peak line flux, semimajor and semiminor axes, and a position angle. The line fluxes were measured by integrating over these spatial 2D Gaussians. In the second ("spectral") approach, we extracted 1D spectra of the [C II] line from the continuum-subtracted cubes. We used apertures that are based on the aforementioned spatial 2D Gaussian profiles, with varying radii ranging $1.5-2 \times \sigma$, where $\sigma$ is the width (i.e., equivalent to standard deviation) of the spatial 2D fitted Gaussian profiles. We then fitted the emission line profiles with a simple model of a single Gaussian, from which we obtained the integrated line flux. The [C II] line fluxes obtained using the two methods are in good agreement, with differences of no more than 0.1 dex for the six quasar hosts (median difference of $0.05 \mathrm{dex}$ ). We eventually chose to adopt the line fluxes measured through the former, "spatial" approach, as it is less sensitive to the low-S/N regions in the outer extended regions of the sources and/or the wings of the line profiles and since it provides more realistic uncertainties. These line fluxes are reported in Table 2.

The best-fit emission line profiles obtained through the latter ("spectral") approach allow us to obtain the [C II] line centers, and therefore [C II]-based redshifts, as well as the line widths (see Tables 2 and 3). We stress that the centers of the [C II] line profiles were treated as free parameters and not constrained to reflect the previously known redshifts of the quasars. We also note that for four of the quasar hosts, the [C II] line is observed near the edge of the spectral band, due to the differences between the redshifts based on AGN-line- and hostISM-related emission regions (see Section 3.2). The line widths we measure for the quasar hosts are in the range FWHM [C II $~ \sim 240-510 \mathrm{~km} \mathrm{~s}^{-1}$, with a tendency for broader lines among the FIR-bright sources (see discussion in Section 3.3). The formal uncertainties on line width measurements are on the order of $10 \%$ (i.e., $\sim 10-40 \mathrm{~km} \mathrm{~s}^{-1}$ ).

\section{Results and Discussion}

\subsection{Source Detection and Identification}

Figure 1 presents the full-scale continuum emission maps of the sources, extending to about 6 ". $^{\prime \prime}(\sim 45 \mathrm{kpc})$ from the quasars' locations, and Figure 2 shows the equivalent [C II] line emission maps. All six quasar hosts are clearly detected in our new ALMA data, both in continuum and in [C II] line emission, with the least significant $[\mathrm{C}$ II] detection having $\mathrm{S} / \mathrm{N} \sim 4.5$ (for J0935; see Table 2). The [C II] spectra of the quasar hosts are shown in Figure 3 (together with the companions we discuss below). We note that the central frequencies of the [C II] lines are shifted, by several hundreds of $\mathrm{km} \mathrm{s}^{-1}$, from the redshifts determined using the quasars' broad UV emission lines (i.e., Hewett \& Wild 2010). We discuss this in more detail in Section 3.2 below.

For one of the FIR-bright sources, J1511, the ALMA data reveal two faint sub-mm-emitting apparent companions, which are detected at the $7-8 \sigma$ level in continuum emission. Their centers are spatially separated from the quasar host by about 2 ". 1 and 3 " 9 , which corresponds to 14 and $25 \mathrm{kpc}$ (see Table 2). These two sources are fainter than the quasar host, in the ALMA continuum data, by at least a factor of 5. Most importantly, we detect significant [C II] line emission from the companion closer to the quasar host, as seen in Figures 2 and 4. The integrated [C II] line flux suggests that the detection is significant at the $\sim 3.5 \sigma$ level. The spectrum we extract for this companion (Figure 3) demonstrates that the line emission, if associated with a redshifted [C II] transition, is shifted by $\sim 75$ $\mathrm{km} \mathrm{s}^{-1}$ from the [C II] line of the corresponding quasar host (see Table 2). We do not detect any significant [C II] line emission from the other, more distant continuum source accompanying J1511. Moreover, we do not detect any other significant emission in the multi-wavelength data available for the $z \simeq 4.8$ quasars, for any of these two companions of J1511. This may suggest that the most distant source seen in the 
Table 2

Spectral Measurements

\begin{tabular}{|c|c|c|c|c|c|c|c|c|c|c|c|c|}
\hline \multirow[t]{2}{*}{ Sub-sample } & \multicolumn{2}{|c|}{ Target } & \multirow{2}{*}{$\begin{array}{l}\text { Cont. Flux } \\
\text { mJy }\end{array}$} & \multirow{2}{*}{$\begin{array}{c}\nu \\
\mathrm{GHz}\end{array}$} & \multirow{2}{*}{$\begin{array}{c}\text { Cont. Size } \\
\square^{\prime \prime}\end{array}$} & \multirow{2}{*}{$\begin{array}{c}F_{[\mathrm{C}} \text { I] } \\
\mathrm{Jy} \mathrm{km} \mathrm{s}^{-1}\end{array}$} & \multirow{2}{*}{$\begin{array}{c}\mathrm{FWHM}_{[\mathrm{C} \mathrm{II]}} \\
\mathrm{km} \mathrm{s}^{-1}\end{array}$} & \multirow{2}{*}{$\begin{array}{c}\nu_{0,[\mathrm{C} \mathrm{II}]} \\
\mathrm{GHz}\end{array}$} & \multirow{2}{*}{$\begin{array}{c}{\left[\mathrm{C} \text { II] } \text { Size }^{\mathrm{a}}\right.} \\
\square^{\prime \prime}\end{array}$} & \multirow{2}{*}{$\begin{array}{c}L_{[\mathrm{C} \mathrm{II}]} \\
10^{9} L_{\odot}\end{array}$} & \multirow{2}{*}{$\begin{array}{l}\Delta d^{\mathrm{b}} \\
\mathrm{kpc}\end{array}$} & \multirow{2}{*}{$\begin{array}{c}\Delta v^{\mathrm{c}} \\
\mathrm{km} \mathrm{s}^{-1}\end{array}$} \\
\hline & ID & comp. & & & & & & & & & & \\
\hline \multirow[t]{5}{*}{ Bright } & J0331 & QSO & $4.3 \pm 0.2$ & 344.47 & $0.18 \times 0.11$ & $5.2 \pm 0.5$ & $495 \pm 31$ & $331.29 \pm 0.006$ & $0.25 \times 0.15$ & 3.58 & $\ldots$ & $\ldots$ \\
\hline & $\mathrm{J} 1341$ & QSO & $18.5 \pm 0.5$ & 346.84 & $0.23 \times 0.21$ & $6.1 \pm 0.8$ & $384 \pm 16$ & $333.41 \pm 0.006$ & $0.47 \times 0.28$ & 4.51 & $\ldots$ & $\ldots$ \\
\hline & $\mathrm{J} 1511$ & QSO & $10.4 \pm 0.3$ & 347.99 & $0.22 \times 0.19$ & $5.3 \pm 0.4$ & $507 \pm 36$ & $334.69 \pm 0.011$ & $<0.39 \times 0.12$ & 3.42 & $\ldots$ & $\ldots$ \\
\hline & & SMG & $1.6 \pm 0.2$ & 347.99 & $0.43 \times 0.11$ & $2.4 \pm 0.7$ & $313 \pm 38$ & $334.61 \pm 0.021$ & $<0.94 \times 0.16$ & 1.53 & 13.9 & +75 \\
\hline & & B & $2.0 \pm 0.3$ & 347.99 & $0.52 \times 0.28$ & $\ldots$ & $\ldots$ & $\ldots$ & $\ldots$ & $\ldots$ & 25.2 & $\ldots$ \\
\hline \multirow[t]{5}{*}{ Faint } & J0923 & QSO & $3.0 \pm 0.1$ & 348.65 & $0.28 \times 0.24$ & $4.1 \pm 0.3$ & $363 \pm 11$ & $336.09 \pm 0.005$ & $0.45 \times 0.34$ & 3.21 & $\ldots$ & $\ldots$ \\
\hline & & SMG & $1.2 \pm 0.3$ & 348.65 & $0.57 \times 0.36$ & $4.1 \pm 0.7$ & $214 \pm 10$ & $335.82 \pm 0.004$ & $0.60 \times 0.31$ & 2.16 & 36.5 & +246 \\
\hline & $\mathrm{J} 1328$ & QSO & $1.7 \pm 0.1$ & 348.74 & $0.40 \times 0.21$ & $3.1 \pm 0.3$ & $221 \pm 14$ & $336.59 \pm 0.008$ & $0.35 \times 0.31$ & 1.63 & $\ldots$ & $\ldots$ \\
\hline & & SMG & $0.7 \pm 0.2$ & 348.74 & $<0.56 \times 0.26$ & $2.0 \pm 0.3$ & $423 \pm 43$ & $337.07 \pm 0.020$ & $0.71 \times 0.44$ & 2.01 & 44.5 & -432 \\
\hline & J0935 & QSO & $1.6 \pm 0.1$ & 347.93 & $0.26 \times 0.16$ & $0.9 \pm 0.2$ & $338 \pm 42$ & $334.49 \pm 0.016$ & $0.44 \times 0.25$ & 0.80 & $\ldots$ & $\ldots$ \\
\hline
\end{tabular}

Notes.

${ }^{a}$ Note that the deconvolved sizes of the [C II] emitting regions are smaller than those reported in Table 1. CLEAN performed with weighting = "briggs."

b Distances between the companions and the quasar hosts, calculated based on the centroids of the ALMA continuum emission, and assuming the redshifts of the quasar hosts' [C II] emission lines.

${ }^{\mathrm{c}}$ Velocity offsets of the accompanying SMGs with respect to the quasar hosts, calculated from the central frequencies of the [C II] emission lines. 
Table 3

Redshifts and [C II] Line Shifts

\begin{tabular}{lcccccc}
\hline \hline $\begin{array}{l}\text { Sub- } \\
\text { sample }\end{array}$ & Target & $z_{[\mathrm{C} \mathrm{II}]}$ & $z_{\text {SDSS }}{ }^{\mathrm{a}}$ & $\begin{array}{l}\Delta v_{\text {SDSS }} \\
\mathrm{km} \mathrm{s}^{-1}\end{array}$ & $z_{\mathrm{Mg} \mathrm{II}}^{\mathrm{b}}$ & $\begin{array}{r}\Delta v_{\mathrm{Mg} \mathrm{II}} \\
\mathrm{km} \mathrm{s}^{-1}\end{array}$ \\
\hline Bright & $\mathrm{J} 0331$ & 4.73678 & 4.73186 & +257 & 4.72890 & +412 \\
& $\mathrm{~J} 1341$ & 4.70030 & 4.68172 & +981 & 4.68944 & +573 \\
& $\mathrm{~J} 1511$ & 4.67850 & 4.67683 & +88 & 4.66988 & +456 \\
\hline Faint & $\mathrm{J} 0923$ & 4.65485 & 4.65001 & +257 & 4.65887 & -213 \\
& $\mathrm{~J} 1328$ & 4.64644 & 4.64998 & -188 & 4.65815 & -621 \\
& $\mathrm{~J} 0935$ & 4.68189 & 4.69920 & -911 & 4.67078 & +588 \\
\hline
\end{tabular}

Notes.

a SDSS-based redshifts taken from Hewett \& Wild (2010).

${ }^{\mathrm{b}} \mathrm{Mg}$ II 22798-based redshifts taken from T11.

ALMA maps of J1511 is a projected companion source, not physically related with the J1511 system. However, given the low probability of observing such a (second) faint sub-mm source within a single ALMA pointing (see below), it may be indeed tracing a faint, low-mass, and/or low-SFR galaxy that is associated with the quasar host. In such a case, the observed properties suggest that it would be a minor merger. This latter interpretation is further justified by our findings of clearly interacting companions, both for J1511 and two of the FIRfaint quasar systems, as described below. In particular, we note that the interacting companions show clear [C II] emission, while the distant companion of J1511 does not, despite having comparable FIR continuum fluxes.

We identify two additional emission sources in the maps of two of the FIR-faint sources, J0923 and J1328-one around each of these quasars - which are clearly detected in the ALMA continuum maps. These sources are detected at significance levels of $\sim 3-4 \sigma$, and have continuum flux densities of 1.2 and $0.7 \mathrm{mJy}$ (for the companions of J0923 and J1328, respectively). The centers of these accompanying sources are located 5".6 and 6!" 8 from the quasars hosts, corresponding to about 36.5 and $44.5 \mathrm{kpc}$. These two accompanying sources show associated [C II] line emission, as seen in the bottom panels of Figure 2 (and also of Figure 4). Similarly to the companion of J1511 described above, the spectra we extract for these two companions (Figure 3) demonstrate that the [C II] line emission is shifted by less than $\sim 450 \mathrm{~km} \mathrm{~s}^{-1}$ from the [C II] lines of the corresponding quasar hosts. We finally note that, similarly to the aforementioned companion of J1511, these two sources are not associated with any other (significant) emission in the multi-wavelength data set we have available for the $z \simeq 4.8$ quasars, particularly the Spitzer data (at observed-frame 3.6 and $4.5 \mu \mathrm{m}$; N14). The (projected) spatial and velocity offsets of all companions are given in Table 2.

Figure 4 presents smaller-scale continuum and line emission maps of all the spectroscopically confirmed systems we identify. The regions where the continuum and line emission is significantly detected are resolved by a few synthesized beams, in all sources. As can be clearly seen, the peaks of the [C II] line emission coincides with the peaks of the dustdominated continuum emission. In the quasars hosts, these peaks of continuum and [C II] emission are also consistent with the locations of the quasars, to within 0 !" 1 , as determined from the SDSS optical imaging.
Before moving on with our analysis of the properties of our quasar hosts and companions and our interpretation of these detections in the context of major galaxy mergers (Section 3.4), we would like to emphasize that the sheer detection of three (and possibly four) faint sub-mm companion sources within our ALMA data are, by itself, highly surprising. The faintest continuum sources in our data (regardless of [C II] detection) have flux densities on the order of $\sim 0.5 \mathrm{mJy}$. Based on recent deep (ALMA) sub-mm surveys (e.g., Karim et al. 2013; Carniani et al. 2015; Aravena et al. 2016a; Fujimoto et al. 2016, and references therein), we would expect on order of $\sim 0.1$ such sources per ALMA pointing (i.e., a circular field of view of $\left.18^{\prime \prime}\right)$. UV-selected, $z \sim 4.8 \mathrm{SF}$ galaxies are yet more rare. The most recent measurements of areal densities in deep fields imply that on the order of 0.01 galaxies with SFR $\sim 100 M_{\odot} \mathrm{yr}^{-1}$ would be observed within a single ALMA band-7 pointing (Stark et al. 2009; Bouwens et al. 2015). Most, but not all such galaxies would have [C II] detections (e.g., Capak et al. 2015; Aravena et al. 2016b). Number counts of purely [C II]-emitting $z \gtrsim 5$ galaxies are highly uncertain, but would probably amount to roughly 0.06 galaxy per each ALMA pointing (e.g., Aravena et al. 2016b). Thus, based on deep (sub-mm) surveys that are designed to detect high-redshift SF galaxies-our robust detection of continuum-emitting companions in the fields surrounding three of the quasar hosts cannot be attributed to chance coincidence. Obviously, the robust detection of [C II] in three of these companions further strengths this conclusion.

We conclude that the host galaxies of all six quasars are clearly detected in the new ALMA data, in both continuum and [C II] line emission. We identify three sub-mm galaxies (SMGs hereafter) accompanying three of the quasar systems-one FIR-bright and two FIR-faint (one SMG accompanying each of these quasars). The companion SMGs are located between roughly 14 and $45 \mathrm{kpc}$ (projected) from the quasars, with relatively small velocity offsets, $|\Delta v|<450 \mathrm{~km} \mathrm{~s}^{-1}$. The three companion [C II]-emitting SMGs-detected solely through the new ALMA data-are therefore physically related to, and interacting with the quasar systems. We finally note that no additional line-emitting sources were found in our ALMA data.

\section{2. [C II] Emission Line Properties}

As clearly seen in Figure 3, the [C II] lines we measure in our sample are often offset toward the lower frequency edge of the observed bands-redshifted with respect to the rest-frame UV emission lines of the quasars. Table 3 compares the redshifts determined from the $[\mathrm{C} \mathrm{II}]$ line to those determined from the Mg II line and also the enhanced SDSS-based redshift determinations published in Hewett \& Wild (2010). The $\mathrm{Mg}$ II line is believed to be among the best redshift indicators for unobscured AGNs, with a scatter of about $200 \mathrm{~km} \mathrm{~s}^{-1}$ compared to the systemic redshift (e.g., Richards et al. 2002; Shen et al. 2016, and references therein). We note that for our $z \simeq 4.8$ quasars the SDSS-based redshift determinations rely solely on the (rest-frame) UV lines Ly $\alpha$ and C IV $\lambda 1549$, both of which may be problematic for the purpose of redshift determinations. The $\mathrm{C}$ IV line is known to present significant blueshifts with respect to systemic redshifts (e.g., Shang et al. 2007; Richards et al. 2011; Shen et al. 2016, and references therein), while the profile of the Ly $\alpha$ line, and particularly the blue wing, is affected by IGM absorption (e.g., Becker et al. 2013). 

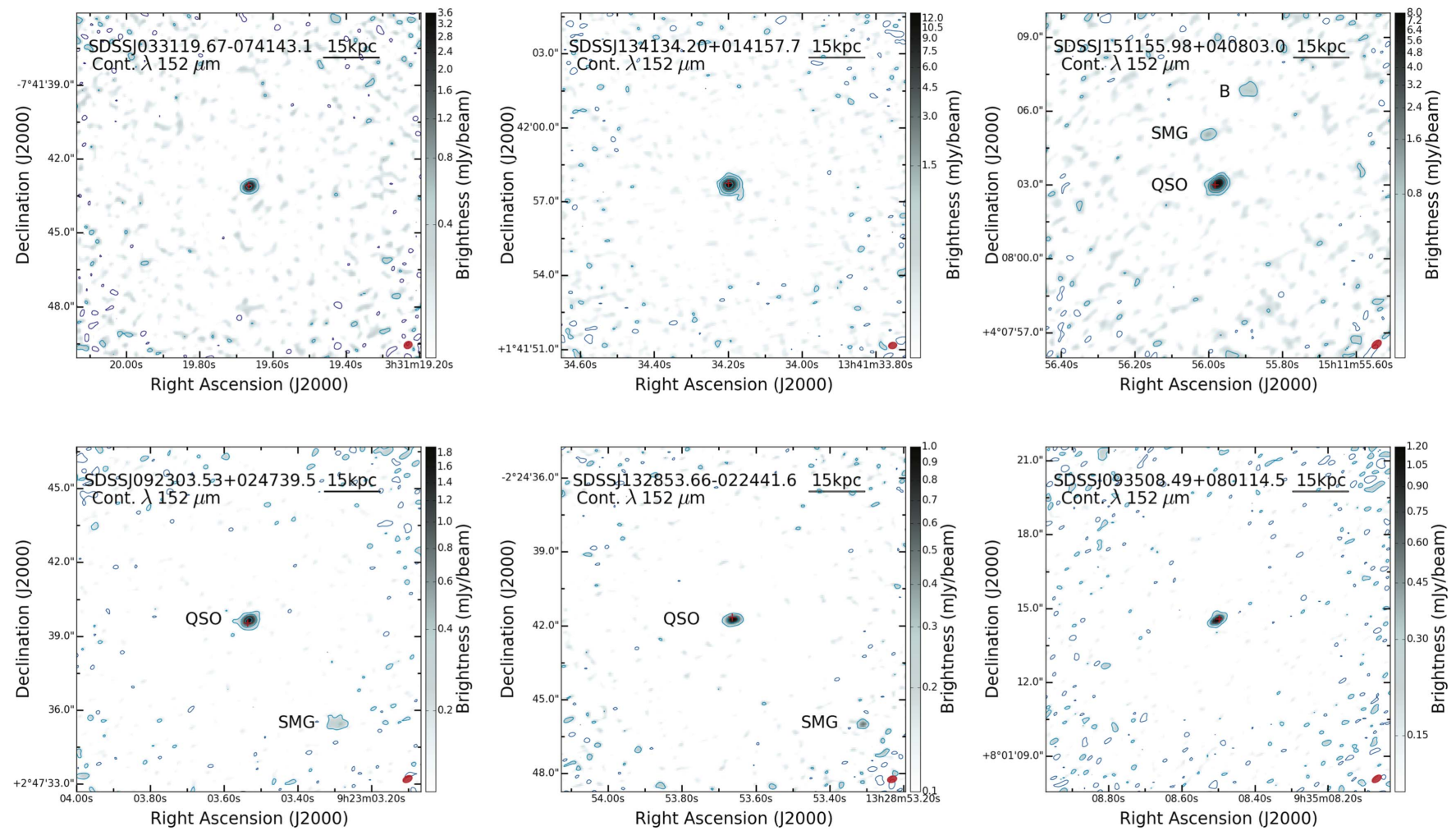

Figure 1. Large-scale continuиm emission maps derived from the new ALMA data for the six quasar systems in our sample: the three FIR-bright sources (top row) and the three FIR-faint sources (bottom row). In each panel, the quasar and any accompanying sub-millimeter galaxies are marked as "QSO" and "SMG," respectively. The gray-scale map shows the continuum emission, determined from the line-free ALMA spectral windows. Cyan and blue contours trace emission levels at different positive and negative significance levels, respectively, with the first contour tracing the region where the continuum emission exceeds $2.5 \sigma$, and consecutive contours plotted in steps of 2.5 $\sigma$. The ALMA beams are shown as red ellipses near the bottom-right of each panel. Red crosses mark the locations of the quasars' optical emission (taken from the SDSS). Interacting companions, i.e., sources that have clear detections of [C II] with redshifts consistent with those of the quasars, are marked as "SMG." The continuum source accompanying J1511 that lacks significant [C II] emission is marked as "B."

All quasar hosts present significant shifts of the [C II] lines, compared to the broad UV emission lines of the quasars. The three FIR-bright hosts and one of the FIR-faint ones (J0935) show [C II] emission redshifted by $\sim 390-580 \mathrm{~km} \mathrm{~s}^{-1}$. Interestingly, the only sources presenting [C II] blueshifts, of $\sim 220$ and $630 \mathrm{~km} \mathrm{~s}^{-1}$, are the two FIR-faint sources, J0923 and J1328, respectively, which have interacting SMGs. Such significant positive velocity shifts of ISM lines, such as [C II], with respect to the quasar's broad emission lines, such as $\mathrm{Mg}$ II-which probe the close vicinity of the SMBHs, are not uncommon in high-redshift quasars. For example, the recent study of Venemans et al. (2016) shows [C II] velocity shifts of $\sim 370-1690 \mathrm{~km} \mathrm{~s}^{-1}$ in a compilation of seven $z>6$ quasars. Willott et al. (2015) found a shift of $\sim 1150 \mathrm{~km} \mathrm{~s}^{-1}$ for one $z \simeq 6$ quasar, but no significant shift for another. Negative velocity shifts of several hundred $\mathrm{km} \mathrm{s}^{-1}$ were also observed among other high-redshift quasars (e.g., Wang et al. 2013; Willott et al. 2013).

Figure 5 shows the $[\mathrm{C}$ II] velocity maps of the six quasar hosts and the three SMGs accompanying J1511, J0923 and J1328. The relatively smooth gradients suggest that the [C II] emission originates from a kiloparsec scale, rotating gas structure, with a rotation axis that coincides with the centers of the galaxies and with the quasars themselves (as shown by the centroid markers). The only obvious outlier is the host of J0935, where the [C II] emission is weaker. For the FIR-bright quasar hosts, the velocity maps reach maximal velocity values of $\left|v_{\max }\right| \simeq 200 \mathrm{~km} \mathrm{~s}^{-1}$, while for the FIR-faint hosts and their accompanying SMGs, the corresponding values are significantly lower, $\left|v_{\max }\right| \simeq 100 \mathrm{~km} \mathrm{~s}^{-1}$. This may suggest that the FIR-bright quasar hosts are more massive, or gas rich, than the FIR-faint ones.

The velocity dispersion maps, presented in Figure 6, show increased velocity dispersions in the centers of all [C II]-emitting systems, reaching $\sigma_{\mathrm{v}} \sim 100 \mathrm{~km} \mathrm{~s}^{-1}$. Given the beam sizes of our ALMA data, this trend is probably mostly driven by the effects of beam smearing, as demonstrated in several detailed studies of the kinematics of high-redshift, sub-mm sources (e.g., De Breuck et al. 2014). A clear signature of this effect is a centrally peaked velocity dispersion, elongated along the minor axis of rotation-which is similar to what is seen in some of our sources (e.g., J1341, J1511, and J0923). Thus, the real underlying central velocity dispersions may be significantly lower than what is seen in Figure 6, resulting in generally more uniform or flatter velocity dispersion profiles, and thus implying rotation-dominated kinematics with $v / \sigma_{\mathrm{v}} \gtrsim 1$. We note that even in such a situation, of $v / \sigma_{\mathrm{v}}$ on the order of a few, the kinematics may be significantly affected by a turbulent component, as demonstrated in several recent studies of resolved ISM kinematics in high-redshift galaxies (e.g., Gnerucci et al. 2011; Williams et al. 2014). With these limitations in mind, we cautiously conclude that the outer parts of the [C II]-emitting regions in all sources are dominated by rotation, with $v / \sigma_{\mathrm{v}}>1$. In particular, this is the case in the outer high-velocity regions of the FIR-bright systems, where one could have expected to see evidence for dispersiondominated gas kinematics in the case these systems were driven by major mergers. 

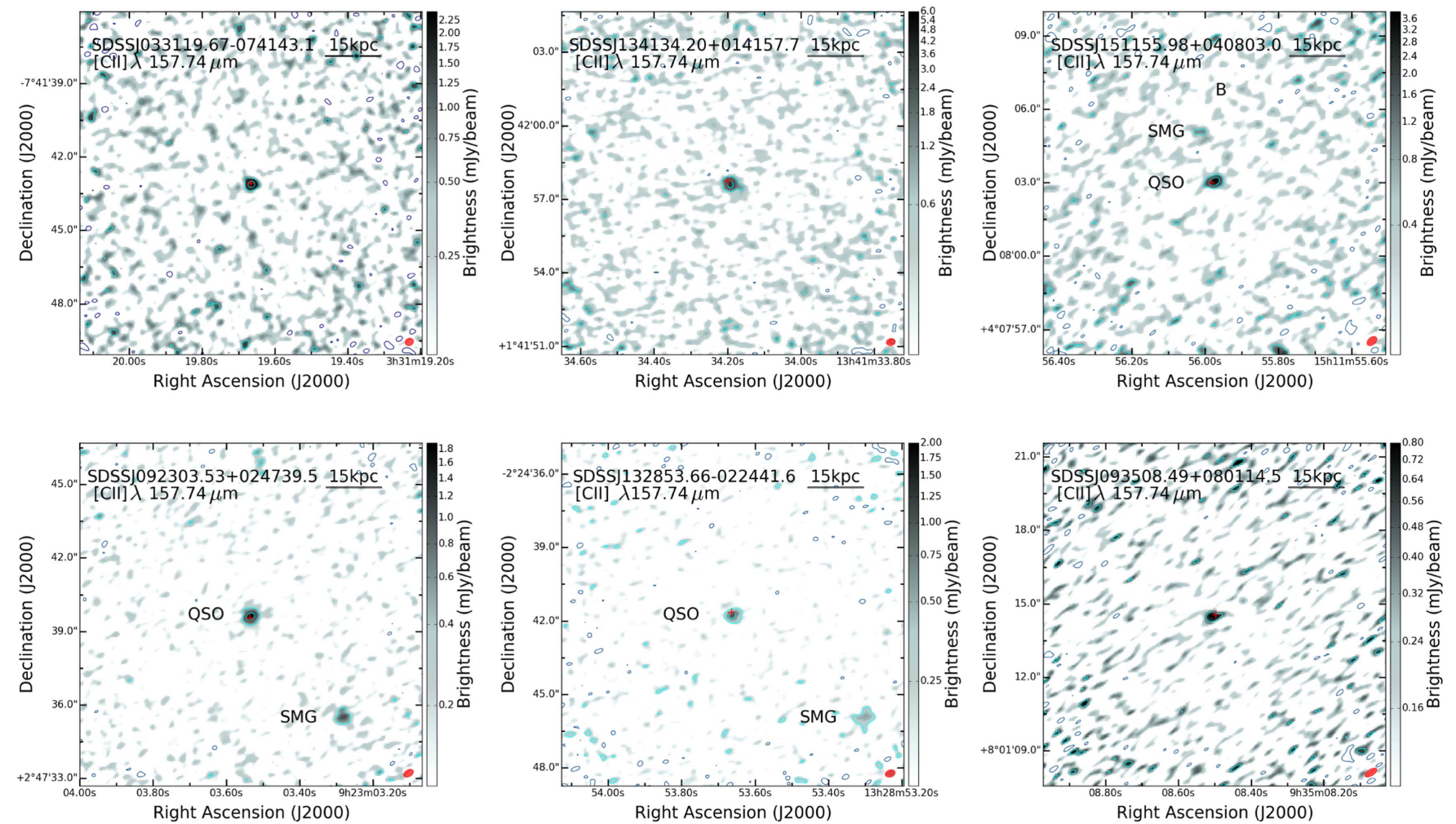

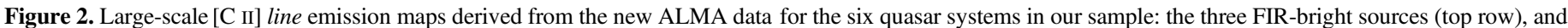

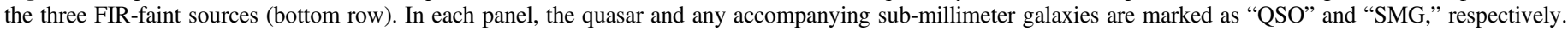

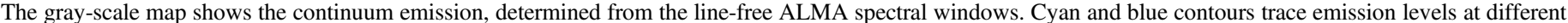

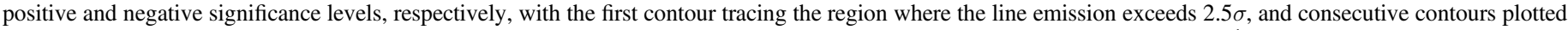

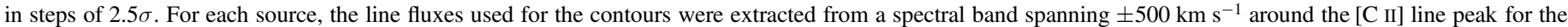

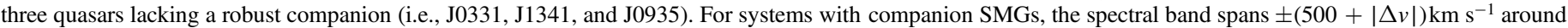

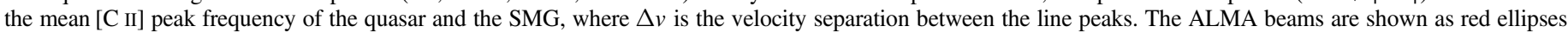

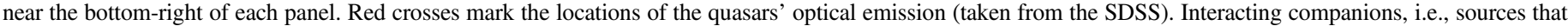

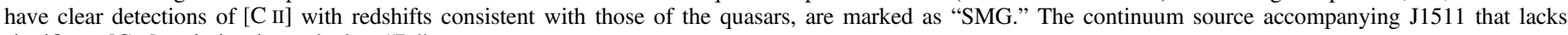
significant [C II] emission is marked as "B."

We next turn to the relative $[\mathrm{C} \mathrm{II}]$ line strength, traced by the ratio between $[\mathrm{C}$ II] line luminosity and the continuum (restframe) FIR luminosity, $L_{\text {[C II] }} / L_{\mathrm{FIR}}$. Figure 7 shows $L_{\text {[C II] }} / L_{\mathrm{FIR}}$ versus $L_{\mathrm{FIR}}$ for the six quasar hosts and the three interacting SMGs in our sample, as well as a large compilation of other galaxies where the [C II] line was detected. The compilation, adapted from Cicone et al. (2015), includes inactive starforming galaxies at low and intermediate redshifts (from Stacey et al. 2010; Díaz-Santos et al. 2013) and SMGs and quasar hosts at $z>4$ (from Maiolino et al. 2009; Ivison et al. 2010; Wagg et al. 2010; Cox et al. 2011; Valtchanov et al. 2011; Swinbank et al. 2012; Venemans et al. 2012, 2016; Carniani et al. 2013; Riechers et al. 2013, 2014; Wang et al. 2013; Willott et al. 2013; De Breuck et al. 2014; Neri et al. 2014). For the purpose of this comparative analysis, we calculated the FIR luminosities assuming a gray-body SED with a dust temperature of $T_{\mathrm{d}}=47 \mathrm{~K}$ and a power-law exponent of $\beta=1.6$, scaled to match the continuum emission of each of the ALMA-detected sources. These scaled SEDs are then used to calculate the integrated luminosity between 42.5-122.5 $\mu \mathrm{m}, L_{\mathrm{FIR}}(42.5-122.5)$. As mentioned in Cicone et al. (2015), the measurements of the sources in the compilation were also scaled, to provide consistent estimates of $L_{\mathrm{FIR}}(42.5-122.5)$. We present a more detailed analysis of the FIR SEDs of our sources in Section 3.3.1 below.
For the quasar hosts, the $L_{\text {[C II] }} / L_{\mathrm{FIR}}$ ratio is in the range of (2.1-9.4) $\times 10^{-4}$, spanning a factor of roughly 4.5. As Figure 7 shows, the FIR-bright and FIR-faint quasar hosts form a trend of decreasing $L_{\text {[C III }} / L_{\mathrm{FIR}}$ with increasing $L_{\mathrm{FIR}}$, although the range of $L_{\text {[C II] }} / L_{\mathrm{FIR}}$ for the two sub-samples overlaps. Since all quasar hosts have comparable $L_{[\mathrm{C} \mathrm{II}}$, this trend of decreasing $L_{\text {[C II] }} / L_{\mathrm{FIR}}$ is mostly driven by the increase in $L_{\mathrm{FIR}}$. The three interacting SMGs follow the same trend, extending to lower $L_{\mathrm{FIR}}$ and higher $L_{\text {[C II] }} / L_{\mathrm{FIR}}$. We further verified that the trend of decreasing line-to-continuum ratio with increasing FIR luminosity is also reflected in the [C II] equivalent widths (EWs; instead of $L_{[\mathrm{CI}} \mathrm{II} / L_{\mathrm{FIR}}$; see, e.g., Sargsyan et al. 2014). The EWs of our quasar hosts are in the range $\mathrm{EW}_{[\mathrm{C} \text { II] }} \simeq 0.2-0.9 \mu \mathrm{m} \quad\left(370-1700 \mathrm{~km} \mathrm{~s}^{-1}\right)$ and follow the same trend with (continuum) FIR luminosity as that found for $L_{\text {[C II] }} / L_{\text {FIR }}$-dropping by about 0.65 dex in EW for a 1 dex increase in (monochromatic) FIR luminosity. The companion SMGs extend this trend to $\mathrm{EW}_{[\mathrm{C} \mathrm{II}]} \simeq 2.4 \mu \mathrm{m}$.

Several recent studies have demonstrated the wide range of possible $L_{[\mathrm{C} \mathrm{II]}} / L_{\mathrm{FIR}}$ in $z \gtrsim 5$ (UV-selected) $\mathrm{SF}$ galaxies, covering $L_{[\mathrm{C} \mathrm{II}]} / L_{\mathrm{FIR}} \sim 0.0002-0.02$-similar to the range observed at lower redshifts (e.g., Capak et al. 2015; Knudsen et al. 2016). Thus, the so-called "[C II] deficit" observed in high-redshift SMGs and quasar hosts is most probably not related to simple observational selection effects in the FIR or sub-mm regime, but rather to the morphology of the SF 

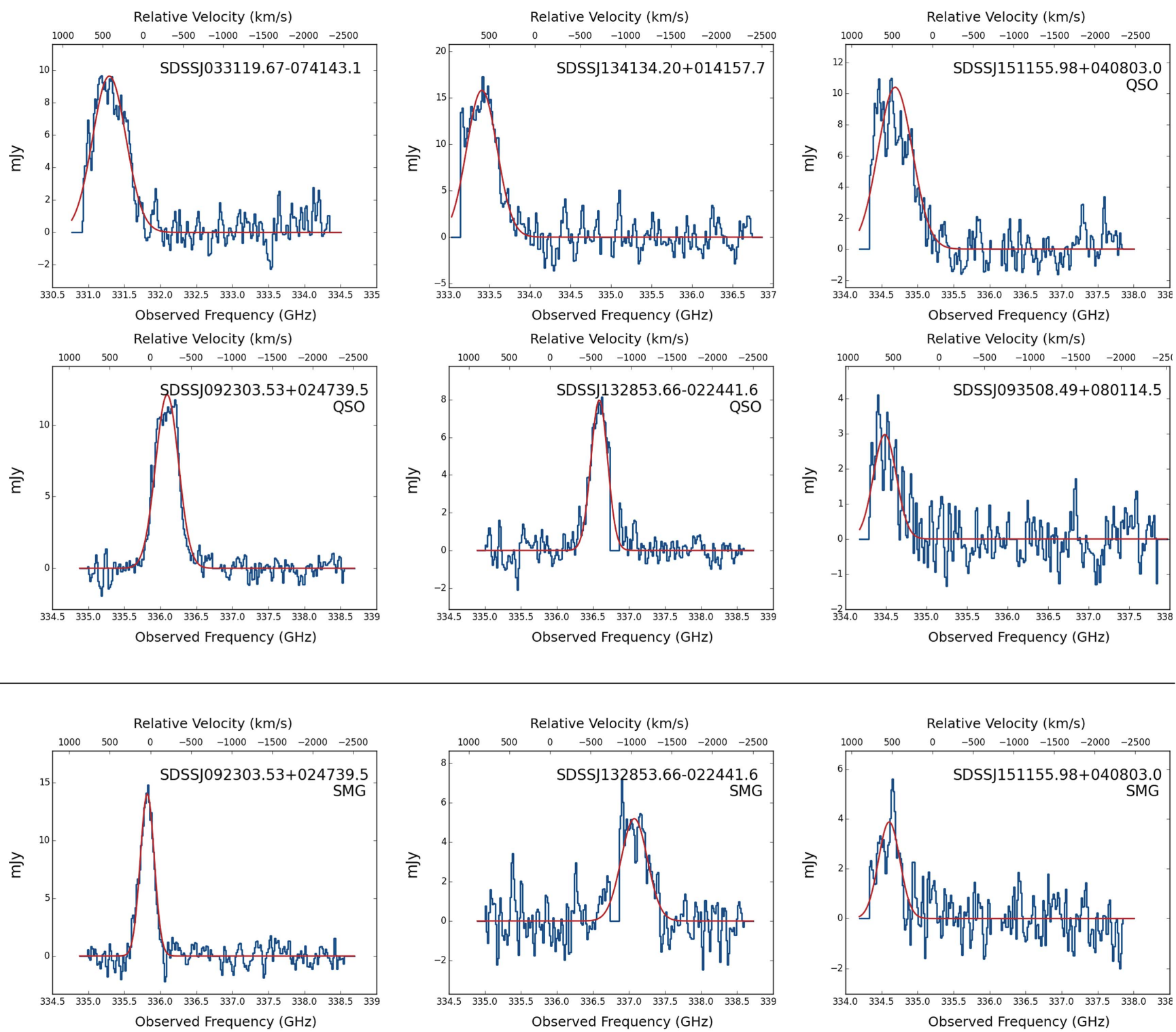

Figure 3. Spectra of the [C II] $\lambda 157.74 \mu \mathrm{m}$ emission line for all the sources with clear line detections: the three "FIR-bright" sources in our sample (top row), the three "FIR-faint" sources (middle row), and the accompanying SMGs (bottom row). The upper $x$-axes denote the velocity offsets with respect to the quasars systemic redshifts, derived from the Mg II broad emission lines (T11). Red lines show the Gaussian fits to the line profiles.

activity. Specifically, high-redshift quasar hosts exhibit more compact starburst-like $\mathrm{SF}$ activity, with $L_{[\mathrm{C} \text { II] }} / L_{\mathrm{FIR}}$ ratios as low as observed in lower-redshift ULIRGs.

Interestingly, our measurements show that the $L_{\text {[C II] }} / L_{\mathrm{FIR}}$ ratio in the three interacting quasar hosts is significantly lower than that found in their companion SMGs. Specifically, we find that the $L_{\text {[C II] }} / L_{\mathrm{FIR}}$ ratio in the quasar hosts of J1511, J0923, and $\mathrm{J} 1328$ is lower by factors of about 1.7, 3, and 2.9, respectively, compared with the companion SMGs. As can be clearly seen in Figure 7, this is consistent with the general trend of decreasing $L_{\text {[C II] }} / L_{\mathrm{FIR}}$ with increasing $L_{\mathrm{FIR}}$, observed for our entire sample of quasar hosts and SMGs. Moreover, this demonstrates that the [C II]-deficit in high-redshift quasar hosts is driven by local properties of the ISM and the UV radiation field within the host galaxies and not by larger scale effects. Indeed, several studies have emphasized that lower $L_{\text {[C II] }} / L_{\mathrm{FIR}}$ ratios are expected to be found in regions with higher SF densities, similar to starbursts-consistent with what is observed (e.g., Díaz-Santos et al. 2013; see discussion in
Cicone et al. 2015). However, the ALMA data for some of our sources do not seem to support this explanation. In particular, the hosts of J1328 and J0935 have virtually identical $L_{\mathrm{FIR}}$ and [C II]-emitting region sizes, but $\left.L_{[\mathrm{C}} \mathrm{II}\right]$ (and therefore $\left.L_{\text {[C II] }} / L_{\mathrm{FIR}}\right)$ in J1328 is twice as high as in J0935. Similarly, the hosts of J0331 and J0923 have very similar $L_{\mathrm{FIR}}$, and $L_{\text {[C II] }} / L_{\mathrm{FIR}}$ in the latter is higher than in the former by merely $30 \%$. However, the $[\mathrm{C} \mathrm{II}]$-emitting region in J0923 is larger by a factor of roughly four than the one in J0331. Thus, our ALMA data do not seem to support a simple scenario where $L_{\text {[C II] }} / L_{\text {FIR }}$ is mainly controlled by the size of the [C II] emitting region, however, higher resolution data are needed to critically address this idea.

\subsection{Host Galaxy Properties \\ 3.3.1. FIR SEDs and SFRs}

The (rest-frame) FIR continuum emission observed within the new ALMA data can be used to estimate the total FIR 

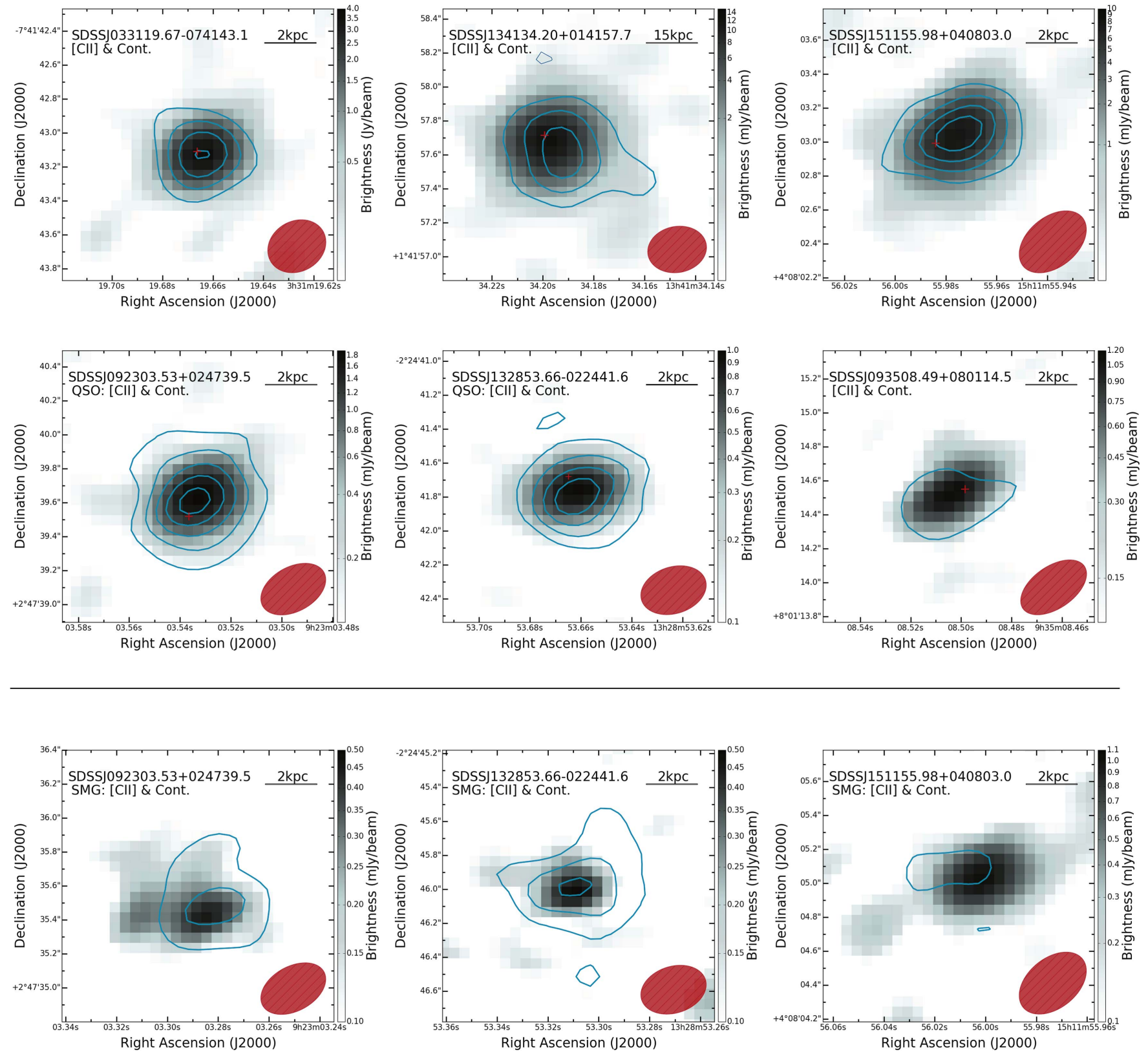

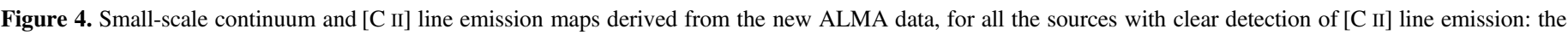

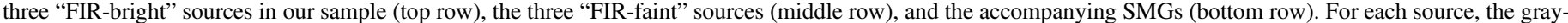

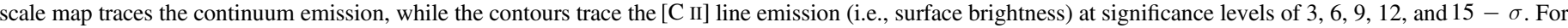

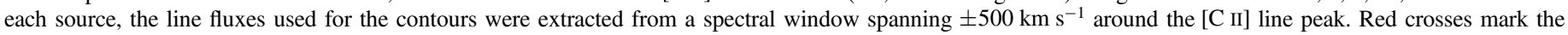
locations of the quasars' optical emission (taken from the SDSS). The ALMA beams are shown as red ellipses near the bottom-right of each panel.

emission and therefore the SFR of the quasar hosts and the accompanying SMGs. This obviously requires additional assumptions regarding the shape of the FIR SED. We expect that the AGN-related contribution to the FIR SED is small, on the level of $10 \%$, at most. This assumption is based on several detailed studies of the mid-to-far-IR SEDs of luminous AGNs across a wide luminosity range (e.g., Schweitzer et al. 2006; Netzer et al. 2007, 2014, 2016; Mor \& Netzer 2012; Rosario et al. 2012; Lutz et al. 2016), although we note that other studies have suggested a higher AGN contribution at FIR wavelengths, particularly at high AGN luminosities (e.g., Leipski et al. 2014; Schneider et al. 2015; Symeonidis et al. 2016; Symeonidis 2017). Given the uncertainties related to the SED shape (see below) and in order to be consistent with other
ALMA studies of high-redshift quasar hosts, we choose to neglect the (small) possible AGN contribution to the FIR SEDs of our sources.

We have reconstructed the FIR SEDs of our sources following two different approaches. First, we adopted the procedure used in several recent studies of FIR emission in high-redshift quasar hosts (e.g., Willott et al. 2015; Venemans et al. 2016, and references therein) and have assumed a graybody SED with dust temperature $T_{\mathrm{d}}=47 \mathrm{~K}$ and $\beta=1.6$. This single temperature dust model is a crude approximation to the more realistic case where dust with a range of temperatures contribute to the observed emission (for a detailed discussion see, e.g., Beelen et al. 2006; Magnelli et al. 2012, and references therein). Second, we have used the grid of FIR SEDs 

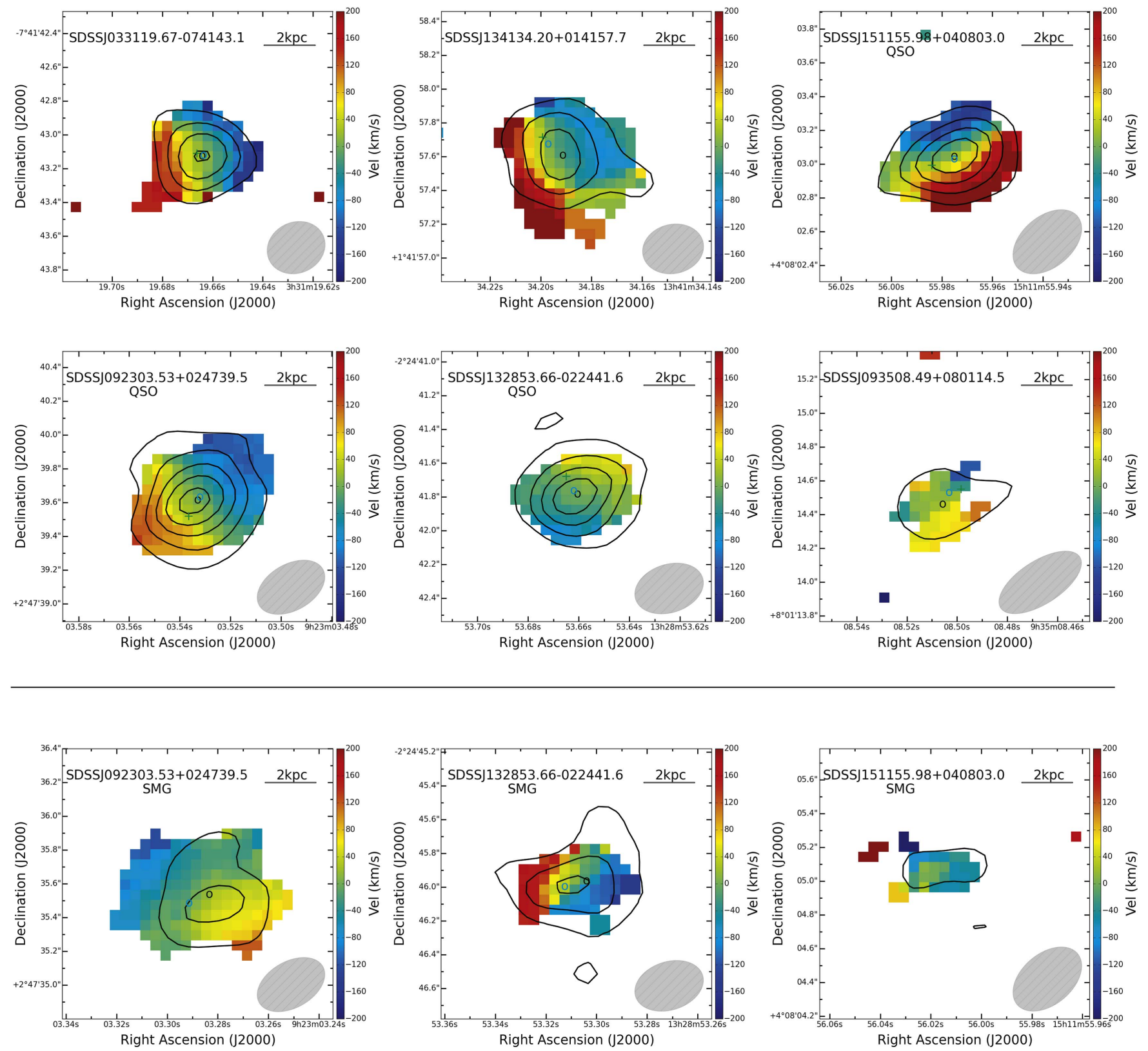

Figure 5. [C II] velocity maps for the three "FIR-bright" sources in our sample (top row), the three "FIR-faint" sources (middle row), and the companion SMGs (bottom row). Black contours trace the [C II] emission line surface brightness at significance levels of 3, 6, 9, 12, and $15-\sigma$. Crosses mark the locations of the quasars' optical emission (taken from the SDSS). Gray circles mark the locations of the peak of the dust (ALMA) continuum emission. Black circles mark the locations of the peak of the [C II] emission. The ALMA beams are shown as hatched gray ellipses near the bottom right of each panel.

provided by Chary \& Elbaz (2001, CE01 hereafter). This grid includes 105 templates spanning a wide range in IR luminosity. As the templates have no free parameters, ${ }^{10}$ we have simply identified the template that best matches the ALMA continuum measurement (i.e., monochromatic luminosity) for each source. This also provides a specific value of total IR (TIR) luminosity, $L_{\text {TIR }}(8-1000 \mu \mathrm{m})$. In the FIR luminosity regime of interest, the uncertainties on the ALMA continuum measurements are typically consistent with, or smaller than, the differences between adjacent FIR templates. The main limitation of this approach is that the template library was constructed to account for low-redshift SF galaxies. However, the small number of

$\overline{10}$ That is, each template SED is unique in shape and scaling. available data points does not warrant the use of other sets of templates.

In Figure 8, we present the ALMA continuum measurements and the Herschel data from N14, along with the two previously mentioned types of FIR SEDs. The low spatial resolution of the Herschel data means that these flux measurements also include the emission from any accompanying continuum sources for the systems where these are resolved by ALMA. We therefore also show in Figure 8 a scaled-down version of the Herschel data points, assuming the relative flux densities of the different neighboring sources follow those of the ALMA measurements. As Figure 8 shows, the gray-body SEDs are generally in good agreement with the previous Herschel data. In particular, in the observed-frame $350 \mu \mathrm{m}$ band (rest-frame wavelength of roughly $60 \mu \mathrm{m}$ ) the ALMA-based SEDs for two of the 

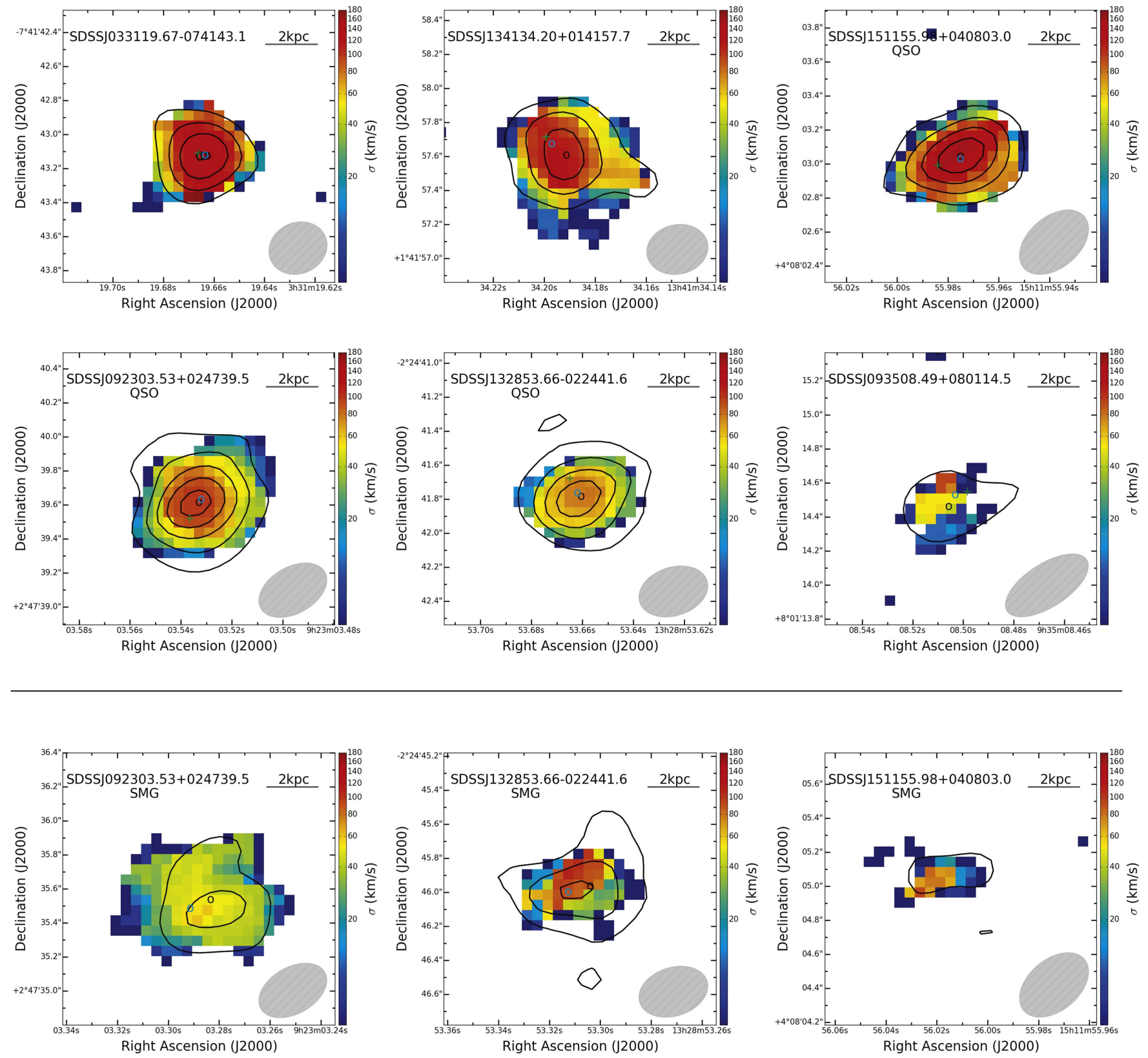

Figure 6. $[\mathrm{C} \mathrm{II}]$ velocity dispersion maps for the the three "FIR-bright" sources in our sample (top row), the three "FIR-faint" sources (middle row), and the companion SMGs (bottom row). Black contours trace the [C II] emission line surface brightness at significance levels of 3, 6, 9, 12, and 15 - $\sigma$. Crosses mark the locations of the quasars' optical emission. Gray circles mark the locations of the peak of the dust (ALMA) continuum emission. Black circles mark the locations of the peak of the [C II] emission. The ALMA beams are shown as hatched gray ellipses near the bottom right of each panel.

FIR-bright systems (J1341 and J1511) differ from the Herschel data by less than 0.05 dex (for either the gray-body or CE01 SEDs). For the third FIR-bright object, J0331, the luminosities expected from the ALMA-based SEDs are significantly lower, by factors of about 2 and 3 , than the fluxes observed with Herschel for the gray-body and CE01 SEDs, respectively. ${ }^{11}$ Since this source has no detectable companions in the ALMA data, we suggest that this discrepancy can only be attributed to the uncertainty on the exact shape of the SED, and perhaps to the limited quality of the Herschel data. Below we investigate how a gray-body SED with a different (higher) temperature may account for this discrepancy.

\footnotetext{
${ }^{11}$ Similarly, the CE01 template that fits the Herschel data alone (as reported in N14) over-predicts the ALMA flux by a factor of 2.4.
}

For the FIR-faint sources, the comparison between the ALMA-based SEDs and the Herschel data is obviously less straightforward, as the Herschel data represent the stacked signal coming from a much larger sample of sources for which the existence of companions that may contribute to the FIR fluxes is unclear. Nonetheless, the agreement between the new ALMA data and the Herschel stacking measurements is respectable. For two of the sources (J1328 and J0935), the ALMA-based gray-body SEDs are consistent within 0.13 dex of the Herschel data (again at observed-frame $350 \mu \mathrm{m}$ ). For the third source (J0923), the gray-body SED over-predicts the luminosity at $350 \mu \mathrm{m}$ by $0.37 \mathrm{dex}$, while the CE01 SED agrees with the Herschel data to within less than 0.1 dex. As a sanity check, if one ignores the scaling factors related to the companions (in J0923 and J1328), that is-assume that the 


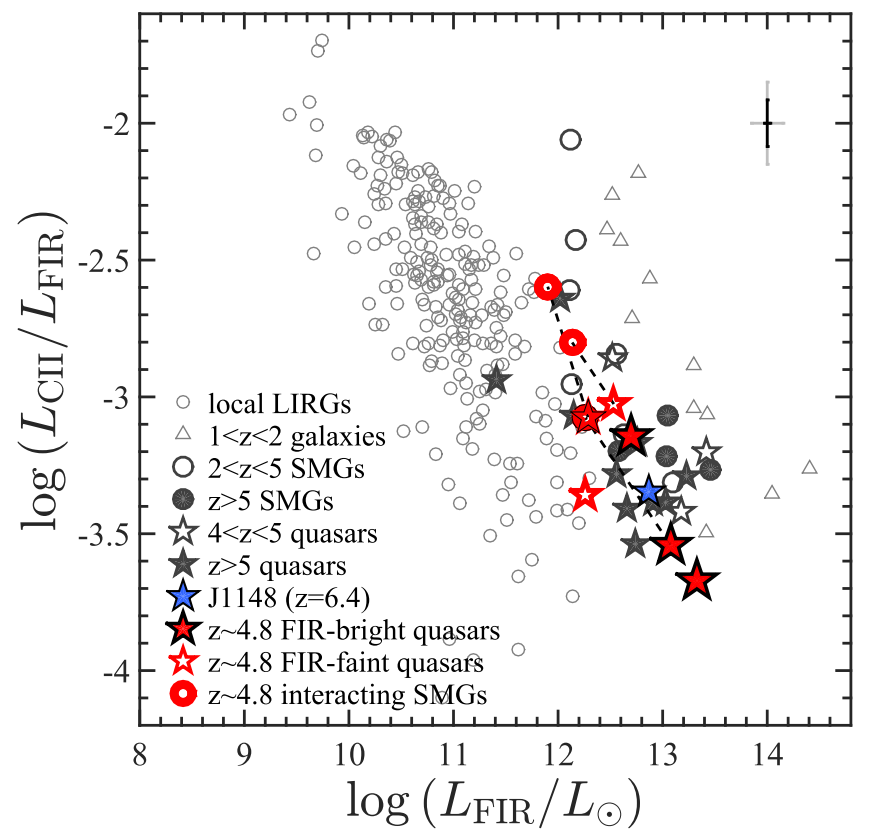

Figure 7. [C II] line luminosity ratio, $L_{[\mathrm{C} \mathrm{II}} / L_{\mathrm{FIR}}$, vs. $L_{\mathrm{FIR}}$ for our sample of $z \simeq 4.8 \mathrm{AGNs}$ and their SMG companions (red symbols) and several reference samples. The quasar hosts under study are marked as red stars (further split to FIR-bright and -faint objects), and their interacting companion SMGs are marked as red circles, connected to the respective quasar hosts by dashed lines. Note that the host galaxy of J1328 and the SMG accompanying J1511 have very similar $L_{[\mathrm{C} \text { II] }}$ and $L_{\mathrm{FIR}}$, therefore their data points overlap in the parameter space we plot. Symbols for reference samples follow those in Cicone et al. (2015, see their Figure 13), including local LIRGs (open circles; Díaz-Santos et al. 2013); star-forming and/or active galaxies at $1<z<2$ (triangles; Stacey et al. 2010); and a compilation of higher-redshift SMGs and quasars, taken from a variety of studies (large circles and stars, respectively; see details in the text). The crosses at the top-right corner illustrate representative measurement (black) and systematic (gray) uncertainties related to $L_{\mathrm{FIR}}$ and $L_{[\mathrm{C} \mathrm{II}]}$. We plot $L_{\mathrm{FIR}}$ systematics of 0.15 dex, reflecting the range covered by the different FIR SEDs we use (i.e., gray-body and template-based; see Section 3.3.1 for details). We note that the ratios we measure for the FIR-bright quasar hosts are among the lowest measured to date, particularly at high redshifts. The interacting quasar hosts have lower $L_{[\mathrm{C} \mathrm{II}]} / L_{\mathrm{FIR}}$ than what is found for the companion SMGs of the same systems.

stacked Herschel signal was not significantly affected by companions, then the differences between the ALMA-based gray-body SEDs and the Herschel data become somewhat smaller.

We have also experimented with both colder and warmer gray-body SEDs, always "anchored" to the new ALMA data. The colder SEDs, with $T_{\mathrm{d}}=40 \mathrm{~K}$ and $\beta=1.5$, identical to those used in N14, systematically under-predict the Herschel data, by $0.1-0.3 \mathrm{dex}$ (at $350 \mu \mathrm{m}$; the discrepancies obviously decrease at observed-frame $500 \mu \mathrm{m})$. Among the warmer SEDs, shown as dotted lines in Figure 8, we note that an extremely warm gray-body with $T_{\mathrm{d}}=65 \mathrm{~K}$ provides a better agreement between the ALMA and the Herschel data for J0331. However, this high temperature is beyond what is typically observed, even among the most luminous FIR sources (e.g., Lee et al. 2013; Magnelli et al. 2014, and references therein). This may suggest that, in this source, the radiation emerging from the vicinity of the SMBH does contribute to heating of galaxy-scale dust, which is not associated with SF regions in the host (e.g., Schneider et al. 2015). We also note that choosing a higher-luminosity template from the CE01 grid would not resolve the discrepancy between the ALMA and
Herschel data for this source, as the highest-luminosity CE01 template that closely matches the Herschel data would significantly over-predict the ALMA continuum measurement. We conclude that both the CE01 and fiducial gray-body FIR SEDs (i.e., with $T_{\mathrm{d}}=47 \mathrm{~K}$ and $\beta=1.6$ ) provide generally good agreement between the ALMA and Herschel data. We therefore chose to use these sets of SEDs in what follows.

We next use the two types of FIR SEDs to calculate the total IR luminosities between $8-1000 \mu \mathrm{m}, L_{\mathrm{TIR}}(8-1000 \mu \mathrm{m})$ for all sources - both quasar hosts and accompanying SMGs. ${ }^{12}$ From these, we estimate SFRs following $\mathrm{SFR} / M_{\odot} \mathrm{yr}^{-1}=$ $L_{\mathrm{TIR}}(8-1000 \mu \mathrm{m}) / 10^{10} L_{\odot}$ (following the assumed Chabrier IMF). Importantly, we note that the agreement between the integrated TIR luminosities obtained with the two types of SEDs is remarkably good. For the six quasar hosts, the $L_{\mathrm{TIR}}$ estimates based on the CE01 template SEDs are lower than those based on gray-body SEDs by merely 0.1 dex (median value). For the three interacting SMGs, the difference is only slightly larger, $\sim 0.13$ dex. The SFRs we obtain for the quasar hosts following this procedure span a wide range of $\sim 260-3040 M_{\odot} \mathrm{yr}^{-1}$ for the gray-body SEDs or $\sim 190-3530 M_{\odot} \mathrm{yr}^{-1}$ for the CE01 SEDs (see Table 4). The SFRs of the FIR-faint sources, $\sim 260-490 M_{\odot} \mathrm{yr}^{-1}$ (or 190-360 $M_{\odot} \mathrm{yr}^{-1}$ using CE01), are in excellent agreement with the value found from stacking analysis performed in N14, using all the Herschel-undetected sources in the parent $z \simeq 4.8$ sample, of roughly $440 M_{\odot} \mathrm{yr}^{-1}$ (see also Netzer et al. 2016 for a slightly lower value). As noted above, the low spatial resolution Herschel stack included the FIR emission from both the quasar hosts and the accompanying SMGs. The total SFRs of the different sub-components in the FIR-faint systems $\left(\sim 680,390\right.$, and $\left.260 M_{\odot} \mathrm{yr}^{-1}\right)$ are, again, consistent with the stacking result. For the FIR-bright sources, the SFRs we derive based on the ALMA data are high, ranging from $\sim 710-3040 M_{\odot} \mathrm{yr}^{-1}$ for the fiducial gray-body FIR SEDs or $\sim 630-3530 M_{\odot} \mathrm{yr}^{-1}$ for the CE01 ones. We note that the lowest SFRs among this sub-sample are those of J0331, where an extremely warm gray-body SED is required to match the Herschel data. Using the $T_{\mathrm{d}}=65 \mathrm{~K}$ gray-body $\mathrm{SED}$ we obtain $\log \left(L_{\mathrm{TIR}} / L_{\odot}\right)=13.35$ and $\mathrm{SFR}=2225 \mathrm{M}_{\odot} \mathrm{yr}^{-1}$. These SFRs are in excellent agreement with those derived in N14, when comparing similar SEDs. The differences between the CE01-based IR luminosities (and therefore SFRs) among the FIR-bright quasar hosts are of 0.1 dex, at most. If we instead consider the gray-body SEDs used in N14, which assumed $T_{\mathrm{d}}=40 \mathrm{~K}$ and $\beta=1.5$, and employ these SED parameters to our ALMA data, then the resulting SFRs are, again, in excellent agreement with the N14 ones for two of the systems (J1341 and J1511). As mentioned above, such a cold SED is inconsistent with the data available for J0331, and the ALMA-based $L_{\text {FIR }}$ for this source is lower than the one obtained in N14 by 0.4 dex.

We conclude that the new ALMA continuum measurements are broadly consistent with the Herschel-based ones (presented in N14) and indicate that all quasar hosts and accompanying SMGs harbor significant SF activity. The exact values of SFR obviously depend on the assumed shape of the FIR SED, but are in good agreement with the ones derived from the Herschel data. The only outlier is the FIR-bright system J0331, where the ALMA-based FIR SED and SFR estimates are found to be

\footnotetext{
${ }^{12}$ We also use these SEDs to calculate $L_{\text {FIR }}(42.5-122.5)$, as mentioned in Section 3.2.
} 


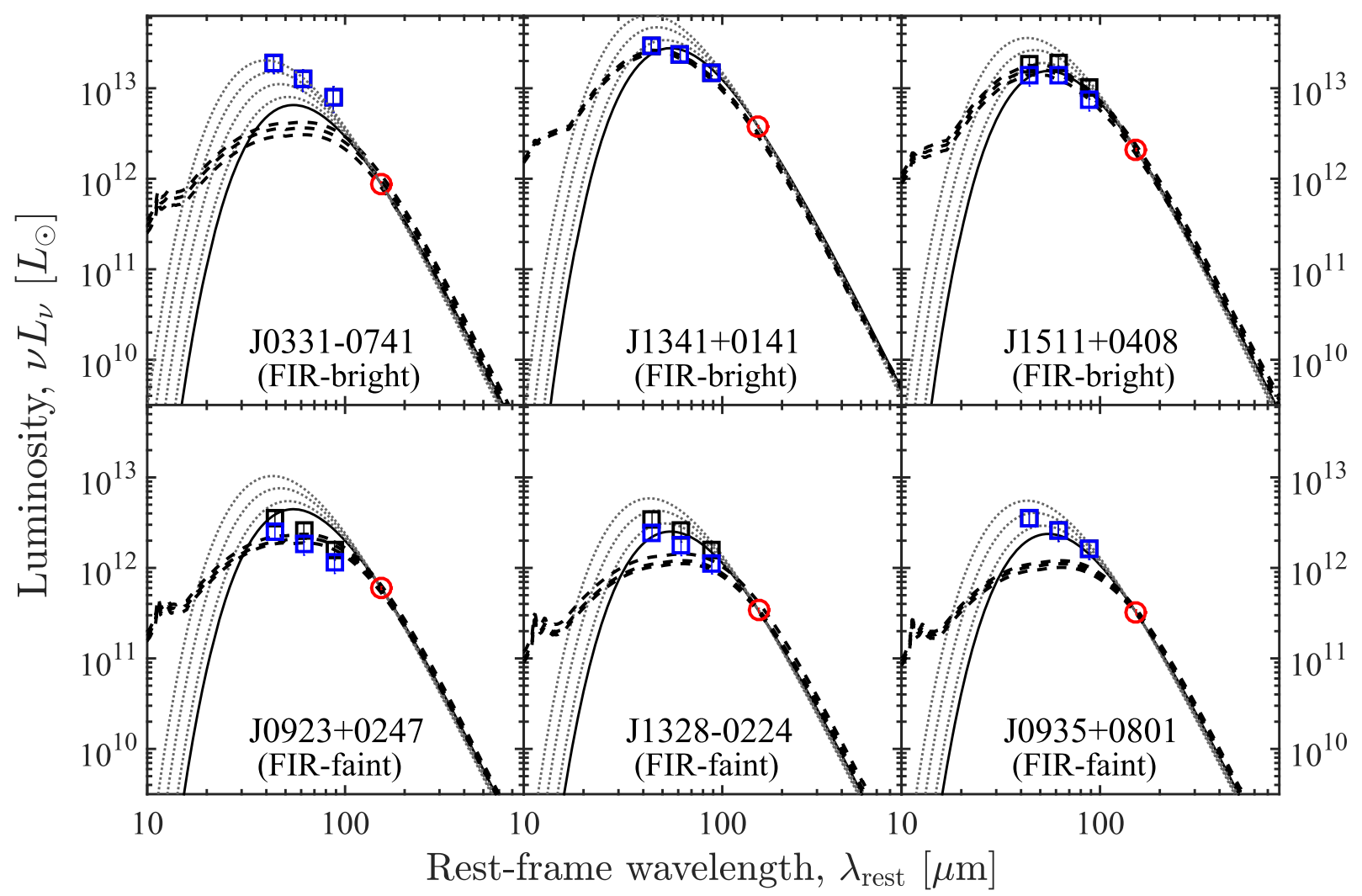

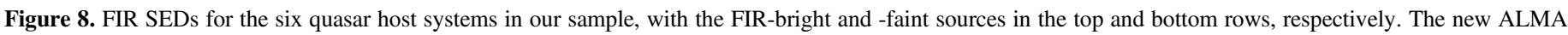

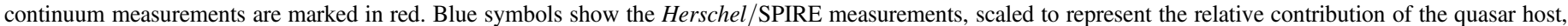

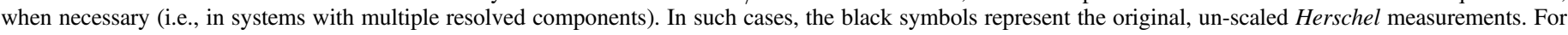

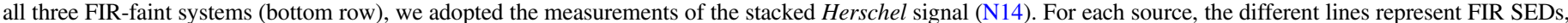

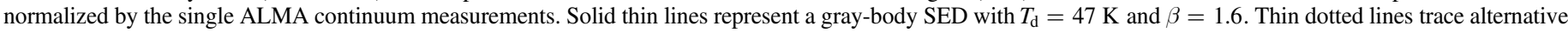

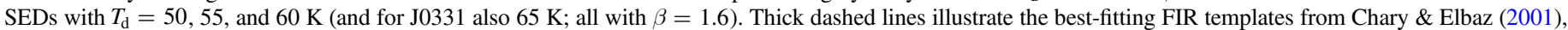
as well as the two adjacent templates (typically separated by $\sim 0.1 \operatorname{dex}$ in $L_{\mathrm{TIR}}$ ).

considerably lower than the Herschel-based ones. Despite these systematic uncertainties, the new ALMA data strongly support the picture that the FIR-bright sources among the T11 sample of $z \simeq 4.8$ quasars have extreme SFRs, exceeding $\sim 1000 M_{\odot} \mathrm{yr}^{-1}$, while the FIR-faint sources have lower, though still intense SFRs, on the order of $\sim 200-400 M_{\odot} \mathrm{yr}^{-1}$.

Most star-forming galaxies are found to populate the socalled "main sequence of star formation" on the SFR- $M_{*}$ plane (SF-MS hereafter). Although this relation is not yet well established at $z>4$, the most recent results from deep surveys suggest a relation of roughly

$$
\mathrm{SFR} \simeq 300\left(\frac{M_{*}}{10^{11} M_{\odot}}\right)^{0.8} M_{\odot} \mathrm{yr}^{-1},
$$

with an intrinsic scatter of $\sim 0.2-0.3$ dex (e.g., Lee et al. 2012; Speagle et al. 2014; Steinhardt et al. 2014). The SFRs we find for the FIR-faint quasar hosts, and for the companion SMGs, are therefore consistent with those of typical massive, highredshift SF galaxies, with $M_{*} \simeq 10^{11} M_{\odot}$. Several recent studies have highlighted the fact that such SFRs can be sustained without invoking major mergers and instead be driven by the accretion of cold gas onto these galaxies (e.g., Bouché et al. 2010; Lilly et al. 2013). On the other hand, it is challenging to account for the extremely high SFRs found for the FIR-bright sources by assuming MS hosts. Such an assumption would require stellar masses in excess of $\sim 5 \times 10^{11} M_{\odot}$. This would imply that the quasar hosts are among the most massive and rarest galaxies ever observed, at any redshift (e.g., Baldry et al. 2012; Ilbert et al. 2013), with number densities on the order of $\Phi \lesssim 10^{-6}-10^{-5} \mathrm{Mpc}^{-3}$ at $z \sim 5$ (e.g., Duncan et al. 2014; Stefanon et al. 2015). In this context, we note that the quasars we study here are among the most luminous in the universe (by selection) and therefore also represent a population of rare objects, with number densities on the order of $\Phi \lesssim 10^{-8} \mathrm{Mpc}^{-3}$ (e.g., Richards et al. 2006; McGreer et al. 2013). Alternatively, it is possible that the FIR-bright sources are hosted in galaxies with masses that are comparable to those of the FIR-faint systems, but located well above the SF-MS. Such systems are typically associated with short periods of intense starburst activity. In particular, SFRs on the order of $>1000 M_{\odot} \mathrm{yr}^{-1}$ are often observed in "classical" (i.e., luminous) SMGs, where they are attributed to late stages of major mergers of massive gas-rich galaxies (see, e.g., the recent review by Casey et al. 2014 and references therein). In the next section we use the available [C II] data to constrain the (dynamical) masses of the quasar hosts, and of their companions, and return to the question of their location in the SFR-mass plane. We discuss the relevance of the major merger interpretation for our sample in Section 3.4. 
Table 4

Galaxy Properties

\begin{tabular}{|c|c|c|c|c|c|c|c|c|c|c|c|}
\hline \multirow[t]{2}{*}{ sub-sample } & \multicolumn{2}{|c|}{ Target } & \multirow{2}{*}{$\begin{array}{c}\log L_{\mathrm{TIR}}{ }^{\mathrm{a}} \\
\left(L_{\odot}\right)\end{array}$} & \multirow{2}{*}{$\begin{array}{c}\log L_{\mathrm{TIR}}{ }^{\mathrm{b}} \\
\left(L_{\odot}\right)\end{array}$} & \multirow{2}{*}{$\begin{array}{c}\mathrm{SFR}^{\mathrm{a}} \\
\left(M_{\odot} \mathrm{yr}^{-1}\right) \\
\end{array}$} & \multirow{2}{*}{$\begin{array}{c}\mathrm{SFR}^{\mathrm{b}} \\
\left(M_{\odot} \mathrm{yr}^{-1}\right)\end{array}$} & \multirow{2}{*}{$\begin{array}{c}\log M_{\text {dyn }} \sin ^{2} i \\
\left(M_{\odot}\right)\end{array}$} & \multirow{2}{*}{$\begin{array}{c}\log M_{\text {dyn }}{ }^{c} \\
\left(M_{\odot}\right)\end{array}$} & \multirow{2}{*}{$\begin{array}{c}\log M_{\mathrm{BH}}{ }^{\mathrm{d}} \\
\left(M_{\odot}\right)\end{array}$} & \multirow[t]{2}{*}{$M_{\mathrm{dyn}} / M_{\mathrm{BH}}$} & \multirow[t]{2}{*}{$\dot{M}_{*} / \dot{M}_{\mathrm{BH}}$} \\
\hline & ID & comp. & & & & & & & & & \\
\hline \multirow[t]{4}{*}{$\overline{\text { Bright }}$} & J0331 & QSO & $12.85^{\mathrm{f}}$ & 12.80 & $715^{\mathrm{f}}$ & 625 & 10.58 & 10.78 & 8.83 & 88 & 32 \\
\hline & $\mathrm{J} 1511$ & QSO & 13.23 & 13.34 & 1696 & 2180 & 10.80 & 10.85 & 8.42 & 264 & 191 \\
\hline & & SMG & 12.42 & 12.28 & 261 & 191 & 10.77 & 10.78 & $\ldots$ & $\ldots$ & $\ldots$ \\
\hline & & $\mathrm{B}^{\mathrm{g}}$ & 12.51 & 12.39 & 326 & 246 & $\ldots$ & $\ldots$ & $\ldots$ & $\ldots$ & $\ldots$ \\
\hline & $\mathrm{J} 1328$ & QSO & 12.44 & 12.31 & 277 & 206 & 10.12 & 10.78 & 9.08 & 50 & 20 \\
\hline & & SMG & 12.06 & 11.86 & 114 & 73 & 10.82 & 11.03 & $\ldots$ & $\ldots$ & $\ldots$ \\
\hline & J0935 & QSO & 12.42 & 12.28 & 261 & 191 & 10.40 & 10.57 & 8.82 & 56 & 16 \\
\hline
\end{tabular}

Notes.

${ }^{a}$ Calculated assuming a gray-body SED with $T_{\mathrm{d}}=47 \mathrm{~K}$ and $\beta=1.6$.

b Calculated from the best-fit template SED of Chary \& Elbaz (2001).

${ }^{c}$ Calculated using the inclination-angle corrections derived from the sizes of the $[\mathrm{C}$ II]-emitting regions.

d Black hole masses taken from T11.

e Calculated assuming the CE01-based SFRs, $\dot{M}_{\mathrm{BH}}=(1-\eta) L_{\mathrm{bol}} / \eta c^{2}$, and $\eta=0.1$.

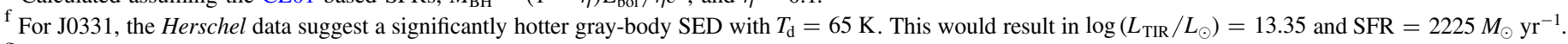

$\mathrm{g}$ The FIR luminosities for the faint companion of J1511 that lacks [C II] detection assume the same [C II]-derived redshift as the quasar host.

\subsubsection{Host and Companion Galaxy Masses}

The [C II] measurements may be used to estimate the dynamical masses $\left(M_{\mathrm{dyn}}\right)$ of the quasar host galaxies and the companion SMGs. For this purpose, we employ the same prescription as used in several recent studies of [C $\mathrm{II}]$ (and $\mathrm{CO}$ ) emission in high-redshift sources, which assumes the ISM is arranged in an inclined, rotating disk (e.g., Wang et al. 2013; Willott et al. 2015; Venemans et al. 2016, and references therein):

$$
M_{\mathrm{dyn}}=9.8 \times 10^{8}\left(\frac{D_{[\mathrm{C} \mathrm{II}]}}{\mathrm{kpc}}\right)\left[\frac{\mathrm{FWHM}[\mathrm{C} \mathrm{II}]}{100 \mathrm{~km} \mathrm{~s}^{-1}}\right]^{2} \sin ^{2}(i) M_{\odot} .
$$

In this prescription, $D_{[\mathrm{C} \text { II] }}$ is the size (deconvolved major axis) of the [C II]-emitting region (as tabulated in Table 2). The $\sin (i)$ term reflects the inclination angle between the line of sight and the polar axis of the hosts' gas disks, in which the circular velocity is given by $v_{\text {circ }}=0.75 \times \mathrm{FWHM} / \sin (i)$. Practically, under the assumption of an inclined disk, the inclination angle is often derived from the (resolved) morphology of the lineemitting region, following $\cos (i)=\left(a_{\text {min }} / a_{\text {maj }}\right)$, where $a_{\text {min }}$ and $a_{\text {maj }}$ are the semiminor and semimajor axes of the [C II] emitting regions, respectively.

Such dynamical mass estimates carry significant uncertainties due to the different assumptions required to derive them and given the kind of data available for our systems. In particular, our ALMA data may not be able to detect the more extended lower surface brightness [C II]-emitting regions, thus underestimating $D_{[\mathrm{C} \mathrm{II}}$, and consequently $M_{\text {dyn }}$. In this sense, the $M_{\text {dyn }}$ estimates derived from our ALMA data would only trace the very central [C II]-emitting regions, on scales of a few kiloparsecs. Faint extended [C II] emission may also affect the inclination corrections, though this would probably be a subtle effect. More importantly, the inclination corrections for marginally resolved extended sources are somewhat sensitive to non-circular beam shapes, as is the case with some of our data. On the other hand, deeper and higher resolution data may also reveal non-rotating ISM components, thus significantly altering the $M_{\text {dyn }}$ estimates. As noted in Section 3.2, the high central velocity dispersions we observe in our sources are most probably driven by the limited spatial resolution of our ALMA data and not by such non-rotating components. Even if the ISM is indeed mostly found in a rotating disk, then determining the underlying (central) velocity dispersion would result in lower $v_{\text {circ }}$, and therefore lower $M_{\text {dyn }}$. Some of these effects are clearly demonstrated whenever increasingly deeper observations were obtained for some $z \gtrsim 5$ sub-mm sources (e.g., Maiolino et al. 2012; Cicone et al. 2015 for J1148 at $z=6.4$; Gallerani et al. 2012 for BRI 0952-0115 at $z=4.4$; and Carniani et al. 2013 for BR 1202-0725 at $z=4.7$ ). A more detailed discussion of these, and other uncertainties related to $M_{\mathrm{dyn}}$ estimates, is also given in Valiante et al. (2014).

Notwithstanding these uncertainties and caveats, we proceed to estimate the dynamical masses of the sources in our sample, using the prescription given in Equation (2). For the quasar hosts, the dynamical masses are in the range of $M_{\text {dyn }} \sin ^{2}(i) \simeq(1-4.4) \times 10^{10} M_{\odot}$ (see Table 4). The three companion SMGs have $M_{\text {dyn }} \sin ^{2}(i)=5.8 \times 10^{10}, 1.57 \times$ $10^{10}$, and $6.6 \times 10^{10} M_{\odot}$ for the SMGs accompanying J1511, J0923, and J1328, respectively. We further derive rough estimates of the inclination angles based on the observed morphology of the [C II] emission in all [C II]-emitting systems (given in Table 2). For the quasar host of J1511 and the accompanying SMG, where the [C II]-emitting regions are not formally resolved, we use the upper limits on $a_{\min }$ and $a_{\max }$. The inclination angles we deduce for our sources are in the range $i \sim 28-80 \mathrm{deg}$. Taking these inclination corrections into account, we obtain dynamical masses of $M_{\text {dyn }} \simeq(3.7-7.5) \times 10^{10} M_{\odot}$ for the quasar hosts, while for the companion SMGs we have $M_{\text {dyn }}=6 \times 10^{10}$, $2.1 \times 10^{10}$, and $10.7 \times 10^{10} M_{\odot}$ (for the SMGs accompanying $\mathrm{J} 1511$, J0923, and J1328, respectively). We find no significant difference between the dynamical masses of FIR-bright and FIR-faint systems. In what follows, we use these inclinationcorrected estimates of $M_{\text {dyn }}$. 
We first note that the dynamical masses of the quasar hosts cover a very narrow range, $\sim(3.7-7.5) \times 10^{10} M_{\odot}$ (i.e., spanning roughly a factor of 2), and five of the six systems have $M_{\text {dyn }} \sim(6-7.5) \times 10^{10} M_{\odot}$ (i.e., spanning less than 0.1 dex). Interestingly, the latter mass is in excellent agreement with the observed "knee" of the stellar mass function in SF galaxies $\left(M^{*}\right)$, which is known to show very limited evolution up to at least $z \sim 3.5$ (e.g., Ilbert et al. 2013; Muzzin et al. 2013). We also note that the dynamical masses of the interacting SMGs differ from those of the corresponding quasar hosts by factors of about $0.85,0.3$, and 1.8 (for the J1511, J0923, and J1328 systems, respectively). Given the uncertainties on our $M_{\text {dyn }}$ estimates mentioned above, these mass ratios are consistent with our interpretation of these interacting systems being major galaxy mergers (see Section 3.4 below).

To complement our estimates of dynamical masses, we also derive rough estimates of the dust and gas masses in our sources. Dust masses are estimated assuming that the FIR continuum fluxes measured from our ALMA data are emitted by optically thin dust, following an SED with $T_{\mathrm{d}}=47 \mathrm{~K}$ and $\beta=1.6$ and further assuming an opacity coefficient of $\kappa_{\lambda}=0.77\left(850 \mu \mathrm{m} / \lambda_{\text {rest }}\right)^{\beta}$ (following Dunne et al. 2000, for consistency with Venemans et al. 2016; see also, e.g., Beelen et al. 2006). The dust masses we derive are in the range $M_{\text {dust }} \sim(0.4-4.8) \times 10^{8} M_{\odot} \quad$ for the quasar hosts and $(0.2-0.4) \times 10^{8} M_{\odot}$ for the companion SMGs. Importantly, the dust masses of the quasar hosts comprise $<1 \%$ of the dynamical masses. This qualitative result is virtually independent of the significant uncertainties involved in the dust mass estimates (due to the assumptions on the SEDs and on $\kappa_{\lambda}$ ). Rough estimates of gas masses can then be inferred by assuming a (uniform) gas-to-dust ratio of 100. These are rather conservative estimates, as several recent studies have shown that the gas-to-dust ratio in high-redshift hosts may be significantly lower (e.g., as low as $\sim 20-60$; Ivison et al. 2010; Banerji et al. 2016, and references therein). For most of the systems, and particularly the quasar hosts, the gas masses comprise $<20 \%$ of $M_{\text {dyn }}$ and reach $\sim 60 \%$ in only one quasar host (J1341). Adopting the aforementioned lower gasto-dust ratios would obviously result in yet lower gas-todynamical mass ratios. We conclude that the $M_{\mathrm{dyn}}$ estimates of our sources are dominated, to a large degree, by the stellar components within the galaxies.

Using our estimates of $M_{\mathrm{dyn}}$ as proxies for $M_{*}$, we again find that all the FIR-bright systems are found well above the SFMS, offset from the relation in Equation (1) by at least 0.5 dex (J0331) and by up to 1.2 dex (J1341). On the other hand, all the FIR-faint quasar hosts, as well as two of the three accompanying SMGs (those of J1511 and J0923), are consistent with the SF-MS, being within about 0.2 dex of the aforementioned relation, which is consistent with the intrinsic scatter associated with it.

\subsubsection{SMBH-host Galaxy Relations}

We now turn to compare the mass growth rates and the masses of the SMBHs powering our quasars relative to those of the stellar populations in their host galaxies.

For the quasar host galaxies, we assume that the mass grows only due to the formation of new stars at a rate determined by the CE01-based SFRs (see Section 3.3.1 above). For the SMBHs, the growth rates are calculated assuming $\quad \dot{M}_{\mathrm{BH}}=(1-\eta) L_{\mathrm{bol}} / \eta c^{2}$, where $L_{\mathrm{bol}}$ is the

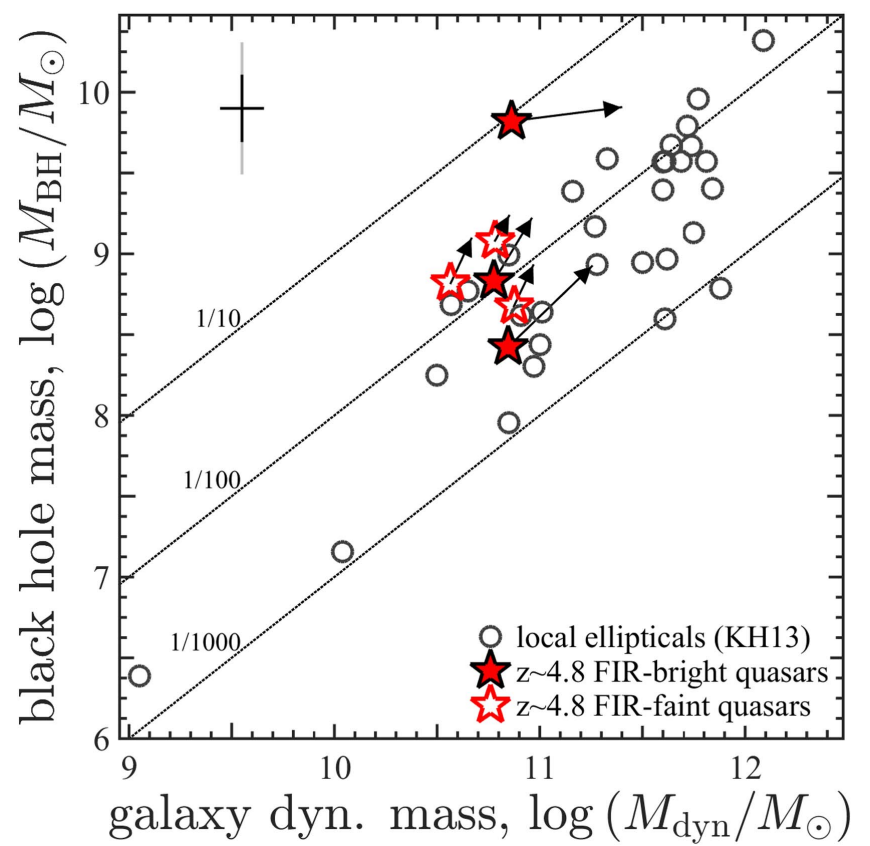

Figure 9. Black hole masses, $M_{\mathrm{BH}}$, vs. host galaxy dynamical masses, $M_{\mathrm{dyn}}$, for our sample of $z \simeq 4.8$ quasars (red stars), compared with a sample of $z \simeq 0$ elliptical galaxies (taken from Kormendy \& Ho 2013, black circles). The dotted diagonal lines trace different constant $\mathrm{BH}$-to-host mass ratios. The crosses at the top-left corner illustrate representative measurement uncertainties (black) on both properties and systematic uncertainties (gray) uncertainties on $M_{\mathrm{BH}}$ (of 0.4 dex). The significant systematic uncertainties on $M_{\text {dyn }}$ (not shown) are more complicated, involving the poorly constrained [C II] emission sizes and inclinations (see Section 3.3.2 for details). Arrows indicate the possible evolution in both the $\mathrm{BH}$ and stellar components, assuming constant mass growth rates over a period of $50 \mathrm{Myr}$. We note that all $\mathrm{BH}$ masses would increase by 0.24 dex if the calibration of Trakhtenbrot \& Netzer (2012) is adopted (see the text for details). Our quasars cover a wide range, with most systems being consistent with the ratio observed in the local universe and some exceeding $M_{\mathrm{BH}} / M_{\mathrm{dyn}} \sim 1 / 100$. The extreme object J1341, which has $M_{\mathrm{BH}} / M_{\mathrm{dyn}} \simeq 1 / 10$ at $z \simeq 4.8$, is expected to evolve toward the locally observed ratio.

bolometric luminosity, estimated from the rest-frame UV continuum emission (see T11), and the radiative efficiency is assumed to be $\eta=0.1$. We find that all systems have $\dot{M}_{\mathrm{BH}} / \dot{M}_{*}>1 / 200$ (see Table 4), with the highest SFR systems J1341 and J1511 having $\dot{M}_{\mathrm{BH}} / \dot{M}_{*} \simeq 1 / 190$ and $1 /$ 120 , respectively. The lower-SFR systems have growthrate ratios as high as $\dot{M}_{\mathrm{BH}} / \dot{M}_{*} \simeq 1 / 30$ (median value). These growth-rate ratios are consistent with those derived in N14 (and in Netzer et al. 2016), which is expected given the consistency between the new ALMA data and the previous Herschel measurements.

As for the mass comparison, we rely on the dynamical mass estimates derived above and the $\mathrm{Mg}$ II-based $\mathrm{BH}$ masses available from T11. These $M_{\mathrm{BH}}$ estimates used the calibration by McLure \& Dunlop (2004). The more recent calibration by Trakhtenbrot \& Netzer (2012) would have increased $M_{\mathrm{BH}}$ by a factor of $1.75(\sim 0.24 \mathrm{dex})$, but we chose not to use it for the sake of consistency with our own previous work and with other samples of $z \gtrsim 5$ quasars (see also Shen et al. 2011; Mejía-Restrepo et al. 2016). Table 4 lists $M_{\mathrm{BH}}$ and the $\mathrm{BH}$-to-host mass ratios, which are in the range $M_{\mathrm{BH}} / M_{\mathrm{dyn}} \sim 1 / 260-1 / 10$. Figure 9 shows our estimates of $M_{\mathrm{dyn}}$ and $M_{\mathrm{BH}}$, along with some similar estimates in local galaxies. For this, we use the subset of elliptical galaxies tabulated in Kormendy \& Ho (2013), for 
which we take $M_{\text {host }}=M_{\text {bulge }} \cdot{ }^{13}$ We note that $M_{\text {bulge }}$ itself, which is indeed the focus of many studies of various types of local galaxies (see an extensive discussion in Kormendy \& Ho 2013), is not accessible with our data.

The mass and growth-rate ratios we derive can be compared to the typical ratios between the masses of galaxies and their central SMBHs, as observed in the local universe. In the $M_{\mathrm{BH}}$ regime of our quasars, these are in the range $M_{\mathrm{BH}} / M_{*} \simeq 1 / 300-1 / 200$ (see Figure 9; e.g., Marconi \& Hunt 2003; Haring \& Rix 2004; Sani et al. 2011; Kormendy \& Ho 2013). As Figure 9 shows, this is broadly consistent only with the lower end of the $M_{\mathrm{BH}} / M_{\mathrm{dyn}}$ range we find in our quasars. Moreover, for four of the six quasar hosts we find $M_{\mathrm{BH}} / M_{\mathrm{dyn}} \gtrsim 1 / 90$, which is significantly higher than the locally observed value. We stress that the BH-to-stellar mass ratios of our sources would be even higher, thus increasing the discrepancy with the local value, recalling the alternative $M_{\mathrm{BH}}$ calibration mentioned above and recalling that $M_{*}<M_{\text {dyn }}$ (Section 3.3.2). At the same time, we have shown that $M_{\text {dyn }}$ itself may be overestimated. Similarly high BH-to-host mass ratios were derived for other luminous $z \gtrsim 5$ quasars using similar data and methods (see, e.g., Walter et al. 2009; Venemans et al. 2012, 2016; Willott et al. 2015, and references therein, but also Lyu et al. 2016). This adds to the evidence for a general trend of increasing $M_{\mathrm{BH}} / M_{\text {host }}$ with increasing redshift, out to $z \sim 2-3$, that is supported by several studies with direct estimates of $M_{\mathrm{BH}} / M_{*}$ (e.g., Decarli et al. 2010; Merloni et al. 2010; Bennert et al. 2011; Bongiorno et al. 2014; Trakhtenbrot et al. 2015, and references therein), as well as indirect arguments (Netzer 2003; Trakhtenbrot \& Netzer 2010; Caplar et al. 2015). It should be noted, however, that the high AGN luminosities of our quasars (i.e., above the break in the quasar luminosity function) may mean that the high $M_{\mathrm{BH}} / M_{\text {host }}$ values we find do not represent the general (active) SMBH population at $z \sim 5$ (see, e.g., Willott et al. 2005; Lauer et al. 2007; Caplar et al. 2015).

Turning back to the $\dot{M}_{\mathrm{BH}} / \dot{M}_{*}$ ratios, the high-SFR systems in our sample are broadly consistent with what is expected if one assumes that SMBHs and their hosts grow "in tandem," obeying the BH-to-stellar mass ratio observed in high-mass systems in the local universe at all times. On the other hand, the high $\dot{M}_{\mathrm{BH}} / \dot{M}_{*}$ ratios found for the rest of the sample (four of six quasars) suggests that in these systems the SMBHs are bound to overgrow the stellar populations. To illustrate the possible evolutionary scenarios for our SMBHs and host galaxies, we plot in Figure 9 the expected mass growth in both the BH and stellar components, assuming the observed (linear) growth rates are sustained for a short period of $50 \mathrm{Myr}$ (as in Venemans et al. 2016). Within this short timespan, most systems will remain broadly consistent with the local range of $M_{\mathrm{BH}} / M_{*}$ ratios. If the SMBHs would stop growing within a comparably short timescale, at about $M_{\mathrm{BH}} \sim 10^{9} M_{\odot}$, then their host galaxies would have to experience only a slightly longer period of SF activity to reach the corresponding local mass ratios $\left(M_{\mathrm{BH}} / M_{*} \sim 1 / 300-1 / 200\right)$. However, if the $\mathrm{BH}$ growth rates are maintained over longer periods, and/or if the SMBHs are instead assumed to be growing exponentially (i.e., at constant $L / L_{\mathrm{Edd}}$, instead of constant $L_{\mathrm{bol}}$ ), then the SMBHs would significantly overgrow their hosts while approaching the highest $\mathrm{BH}$ masses known, $M_{\mathrm{BH}} \sim 10^{10} M_{\odot}$, and moving away

\footnotetext{
${ }^{13}$ Excluding NGC 4486B.
}

from the local BH-host relation. In this mass regime, the corresponding local mass ratios are on the order of $M_{\mathrm{BH}} / M_{*} \sim 1 / 100$, which would still require significant SF activity in our objects.

Our conclusions regarding the relative growth rates are in line with the recent results of Netzer et al. (2016). This study used Herschel data for a large sample of some of the most luminous quasars at $z \gtrsim 2$ (including all of our sources) and found that the vast majority of systems has $\dot{M}_{\mathrm{BH}} / \dot{M}_{*} \gtrsim 1 / 100$, while a small fraction had $\dot{M}_{\mathrm{BH}} / \dot{M}_{*} \sim 1 / 200-1 / 100$. Assuming that the most luminous quasars at $z \gtrsim 2$ form a continuously evolving population, the Netzer et al. (2016) study also suggested that the epoch of fast SMBH growth traced by our sources would extend to $z \sim 2-3$, perhaps at low duty cycles (see T11), while the intense SF activity seen in some of the quasar hosts may decrease shortly after $z \sim 5$. In the context of the $z \simeq 4.8$ quasars we study here, this may indeed mean that our SMBHs would reach the high end of the known $M_{\mathrm{BH}}$ range, with $M_{\mathrm{BH}} \sim 10^{10} M_{\odot}$. The (decreasing) SF activity is still required to suffice to reach $M_{\mathrm{BH}} / M_{*} \sim 1 / 100$.

We finally highlight the exceptional properties of the J1341 system, which has a high of $M_{\mathrm{BH}}=6.6 \times 10^{9} M_{\odot}$, an extremely high BH-to-host mass ratio of $M_{\mathrm{BH}} / M_{*} \sim 1 / 10$, and a low mass-growth ratio of $\dot{M}_{\mathrm{BH}} / \dot{M}_{*} \sim 1 / 190$. All this suggests that the SMBH is approaching its final mass, while the host galaxy is forming stars at an intense rate and the system would likely expected to approach the high mass end of the $z \sim 0$ mass ratio (see Figure 9). This is similar to the over-massive BH CID-947, recently identified at $z \simeq 3.3$, and speculated to have experienced an earlier episode of fast Eddington-limited growth to reach the observed $M_{\mathrm{BH}} \simeq 7 \times 10^{9} M_{\odot}$ and $M_{\mathrm{BH}} / M_{*} \simeq 1 / 10 \quad$ (Trakhtenbrot et al. 2015). ${ }^{14} \mathrm{~J} 1341$ may therefore be illustrative of the scenario of early fast SMBH growth to the highest known mass well before $z \sim 4.5$, with somewhat longer timescale stellar growth, to eventually reach $M_{\mathrm{BH}} / M_{*} \sim 1 / 100$.

\subsection{Major Mergers among Hosts of Fast-growing SMBHs}

Given the results of Herschel analysis available prior to the new ALMA observations, the naive expectation for the sample under study was that the FIR-bright sources are powered by major mergers between gas-rich galaxies, while the FIR-faint sources are evolving secularly or, perhaps, are related to a later evolutionary phase, where the accreting SMBHs may have already affected the SF in their hosts (see N14 for a detailed discussion). Moreover, there is strong evidence that the occurrence rate of mergers among AGNs increases with increasing AGN luminosity and redshift (Treister et al. 2012). Based on these trends, a high occurrence rate of mergers, in excess of $50 \%$ and perhaps as high as $80 \%$, is expected for our $z \simeq 4.8$, high $-L_{\mathrm{AGN}}$ (and high $-L / L_{\mathrm{Edd}}$ ) quasars. The new highresolution ALMA data allow us to critically revisit these ideas. The small size of our sample naturally limits the scope of our interpretation, however, we note that most of the previous studies addressing these questions at comparably high redshifts included yet fewer objects and/or relied on lower-quality data.

At face value, our new ALMA data clearly show that a significant fraction of $z \simeq 4.8$ luminous quasars- $50 \%$ in our

\footnotetext{
${ }^{14}$ It is, however, worth bearing in mind that the two systems (J1341 at $z \simeq 4.8$ and CID-947 at $z \simeq 3.3$ ) are drawn from parent samples of markedly different number densities.
} 
small sample-are interacting with companion galaxies of comparable mass, thus supporting the idea that major mergers are a dominant driver of the intense $\mathrm{BH}$ and SF activity. Moreover, we note that this may constitute a lower limit on the real fraction of interacting quasar hosts, when considering the possibility that additional companions are locate just outside of the ALMA fields (i.e., separated by $\gtrsim 50 \mathrm{kpc}$ ); that additional close companions are too faint to be detected in our ALMA data (i.e., have SFR $\ll 100 M_{\odot} \mathrm{yr}^{-1}$ ) and/or that some of the isolated quasars are actually in the final stages of a merger with interaction signatures that can only be detected with higher resolution data.

However, the properties of the SMBHs in our sample, their hosts and companion galaxies under study, highlight several shortcomings of the simplistic merger-driven growth scenario. First, two of the three quasars with robust detections of physically interacting companions (i.e., SMGs) are actually among the FIR-faint sources, while only one of the FIR-bright sources has an interacting companion (J1511). Second, the velocity maps of all the FIR-bright systems, and particularly those that lack companions, show evidence for ordered rotation, around an axis that coincides with the centroids of the host galaxies (and quasars' locations; see Section 3.2 and Figure 5). The coincidence of the region of zero velocity with the centroids of the SF activity in the hosts suggests that the redshifted and blueshifted [C II] emitting regions are not tracing (smaller) coalescing galaxies. Moreover, as noted in Section 3.2, the outer parts of the ISM in the FIR-bright systems appear to be rotation dominated. This is also seen among the FIR-faint systems, although to a lesser extent.

We also note that the speculation made in N14 that the FIRfaint systems are found in a later evolutionary stage is disfavored by the new ALMA data, as (two of) the FIR-faint systems are seen to be in a rather early stage of a major merger. Their lower SFRs will therefore likely increase as the interacting galaxies coalesce. In principle, a possible interpretation for our new ALMA data may have been that the SMBH activity in the FIR-faint sources is driven by the "first passage" of the interacting quasar hosts with their SMG companions. Indeed, the high SFRs of the interacting galaxies in the J1511 system may be indicative of what the FIR-faint systems would undergo in later stages of the interaction. However, most simulations of major mergers suggest that the SMBH would produce a luminous quasar, with high $L / L_{\text {Edd }}$, at the final coalescence phase, and not in the first passage phase. These same simulations also suggest that the first passage enhances SFR in the interacting hosts, to levels comparable to those found in the later, final coalescence phase (see, e.g., Blecha et al. 2011; DeBuhr et al. 2011; Sijacki et al. 2011; Van Wassenhove et al. 2012, but also Volonteri et al. 2015 and Gabor et al. 2016). Our sample, on the other hand, shows similarly intense SMBH growth, with $L_{\mathrm{AGN}} \simeq 10^{47} \mathrm{erg} \mathrm{s}^{-1}$ and $L / L_{\text {Edd }} \sim 0.5-1$, for both high- and low-SFR quasar hosts and among systems with and without an interacting companion.

Any direct comparison with merger simulations is further complicated by the possibility that the companion SMGs we detect at larger separations of $\sim 40 \mathrm{kpc}$ may have already experienced a much closer passage to the quasar hosts and are observed close to their apocenter. The intense SMBH growth may have been triggered during this pericenter passage, when tidal forces were maximal. In this context, we note that any observations of interacting galaxies would be biased toward large separations, due to the longer periods spent at increasingly larger separations. Indeed, an inspection of several of the aforementioned simulations suggests that the interacting galaxies are separated by $\gtrsim 20 \mathrm{kpc}$ for over $80 \%$ of the simulated merger. First and second passages are extremely short, taking up $\sim 5 \%$ of the time. ${ }^{15}$

We therefore conclude that our ALMA data provide compelling evidence for significant galaxy-galaxy interactions (major mergers) in some, but not all, quasar hosts. Moreover, the links between these interactions and the intense accretion onto the SMBHs remains unclear. For the FIR-bright sources, we caution that even with the new ALMA data, we cannot completely disprove the possibility that all these systems are indeed observed in the advanced stages of a major merger. In particular, the evidence for rotation in the ISM of some quasar hosts does not by itself disprove a merger scenario, as several studies of low-redshift mergers (i.e., ULIRGs) have identified nuclear structures of rotating molecular gas, on scales of a few kiloparsecs (see, e.g., Downes \& Solomon 1998; Tacconi et al. 1999; Ueda et al. 2014 and the discussion in Hodge et al. 2012). Simulations of (low-redshift) major mergers have demonstrated that such gas disks may indeed form several hundreds of Myr after the "peak" of the merger, once the coalesced nucleus has relaxed (e.g., Barnes 2002; Springel \& Hernquist 2005). On the other hand, the interacting companions we identify among the FIR-faint systems cannot be related to the SF and SMBH activity in a straightforward way, given their large separations. The only way in which all the quasars in our sample can be explained as triggered by major merger is if the FIR-bright systems that lack an interacting companion (namely J0331 and J1341) are observed in the final coalescence stage, while the two interacting FIR-faint quasar hosts have already experienced a close first passage that triggered the SMBH growth and are observed close to their apocenter. Even this scenario fails to account for one of the FIR-faint systems (J0935), which has no companions out to $\sim 50 \mathrm{kpc}$.

Several sub-mm studies published in recent years have probed the existence of SF galaxies that are accompanying, or indeed interacting with, the hosts of $z \gtrsim 4.5$ quasars. A prominent example is the interacting system BR1202-0725 at $z=4.7$, which was shown to consist of a pair of interacting SMGs, separated by $25 \mathrm{kpc}$, one of which hosting a luminous quasar, with an additional (marginal) detection of a faint AGNs in the other one (Iono et al. 2006; Wagg et al. 2012; Carniani et al. 2013). However, such close interactions are found to be quite rare among high-redshift quasars. Several deep optical imaging campaigns in the fields around the known $z \simeq 6$ quasars provide no or little robust evidence for physically associated companions (e.g., Willott et al. 2005). Moreover, the sub-mm (ALMA and PdBI) studies published for most of the known $z \gtrsim 6$ quasars (by Wang et al. 2013; Willott et al. 2013, 2015; Venemans et al. 2016)—which could have detected companions with SFRs comparable to those we find at separations of up to $\sim 100 \mathrm{kpc}$-report no such companions.

Taken at face value, this may suggest a fast increase in the occurrence of mergers among luminous quasar hosts between $z \simeq 6.2$ and $z \simeq 4.8$ - that is, within $\sim 350$ Myr. We note,

\footnotetext{
${ }^{15}$ It is worth noting that many simulations are set up in a way that will lead to a merger within a relatively short time, due to computational limitations. The fraction of time spent at large separations, on the order of $\sim 40 \mathrm{kpc}$, may therefore be even higher.
} 
however, that this discrepancy may be driven by the limited sensitivity and/or limited spatial resolution of the available sub-mm (ALMA) data for some $z \simeq 6.2$ quasars (e.g., $\sim 4 \mathrm{kpc}$ in Wang et al. 2013). Additional deep observations of these $z \simeq 6.2$ sources at sub-kiloparsec resolution may indeed resolve this discrepancy.

To conclude the discussion of major mergers, we recall that our SMBHs - as well as the other $z \gtrsim 5$ quasars mentioned above - had to grow continuously and at high rates since very early epochs to account for their high masses (see T11, and references therein). Given the number of interacting companions we find and their separations from the quasars' hosts, it seems unlikely that this kind of mergers can be the only driver of such a prolonged period of fast growth. All of the above suggests the epoch of fastest growth of the most massive BHs is driven, at least in part, by mechanisms that are not related to major mergers - such as direct accretion of IGM gas (e.g., Dekel et al. 2009; Di Matteo et al. 2012; Dubois et al. 2012), minor mergers, and/or galaxy-scale instabilities of the gas or stellar components (e.g., Springel et al. 2005; Bournaud et al. 2011, 2012).

Finally, we note that even if the companion SMGs we find are not directly related to the fueling of the fast-growing $z \simeq 4.8 \mathrm{SMBHs}$, their presence seems to support the idea that rapid early $\mathrm{BH}$ growth preferentially takes place in dense largescale environments (e.g., Di Matteo et al. 2008; Sijacki et al. 2009; Dubois et al. 2012; Costa et al. 2014). To date, such evidence has been highly elusive, with several observational campaigns looking for over-densities of (rest-frame) UVbright galaxies around luminous high-redshift quasars yielding highly ambiguous results (see, e.g., Overzier et al. 2006; Kim et al. 2009; Utsumi et al. 2010; Husband et al. 2013 for several examples of such over-dense environments, but also Willott et al. 2005; Bañados et al. 2013; Simpson et al. 2014 and some of the systems in Kim et al. 2009 for the contrary). Our analysis demonstrates that such studies may be significantly biased against dust-obscured high-redshift SF galaxies, thus underestimating the real (over-)density of galaxies around highredshift quasars. Indeed, the three interacting companions we identify were not identified in the Spitzer images of the respective quasars (N14). A complete census of the cosmic environments of high-redshift, fast-growing SMBHs would therefore require a multi-wavelength approach, covering scales of up to a few arc-minutes, and in spectral regimes that are not affected by dust obscuration. This can be done, for example, using compact sub-mm arrays.

\section{Summary and Conclusions}

We have presented ALMA band-7 observations of six luminous quasars at $z \simeq 4.8$, drawn from a sample of 40 , UVselected SDSS quasars with a wealth of supporting multiwavelength data. The data probe the rest-frame far-IR continuum emission that arises from cold dust, heated by SF in the host galaxies of the quasars, as well as the [C II] $\lambda 157.74 \mu \mathrm{m}$ emission line that originates from the cold phase of the hosts' ISM. The ALMA observations resolve the continuum- and line-emitting regions on scales of $\sim 2 \mathrm{kpc}$. Our main findings are as follows.

1. All quasar hosts are clearly detected and resolved, in both continuum and [C II] line emission. The continuum emission suggests intense SF, with the FIR-bright sources reaching $\sim 1000-3000 M_{\odot} \mathrm{yr}^{-1}$, consistent with Herschel observations of these systems. The quasar hosts exhibit evidence for massive, rotation-dominated gas structures.

2. Three quasar hosts - one FIR-bright and two FIR-faint systems-are accompanied by spectroscopically confirmed, interacting companions, with separations in the range $\sim 14-45 \mathrm{kpc}$ and within $|\Delta v| \lesssim 450 \mathrm{~km} \mathrm{~s}^{-1}$. The companions themselves are forming stars at rates of a few hundred $M_{\odot} \mathrm{yr}^{-1}$, slightly lower than the quasar hosts with which they interact.

3. The remaining quasar hosts-two FIR-bright and one FIR-faint-lack significant companions. This, combined with the evidence for rotation, may suggest that processes other than major mergers are driving the significant SF activity and fast SMBH growth in these systems.

4. The dynamical masses of the quasar hosts, estimated from the $[\mathrm{C}$ II] lines, are within a factor of $\sim 3$ of the masses of the interacting companions, supporting our interpretation of these interactions as major mergers.

5. The [C II]-based dynamical masses also show that the FIR-faint systems are consistent with the "main sequence" of star-forming galaxies, while the FIR-bright systems are located above it.

6. Compared with the $\mathrm{BH}$ masses, the [C II]-based dynamical host masses are generally lower than what is expected from the locally observed $\mathrm{BH}$-to-host mass ratio. In some of the systems, this discrepancy may grow further, given the high accretion rates of the SMBHs.

7. The $L_{[\mathrm{C} \text { II] }} / L_{\text {FIR }}$ ratios in the quasar hosts are consistent with those found in other $z \gtrsim 5$ quasar hosts and SMGs and follow the observed trend of declining $L_{\text {[C II] }} / L_{\text {FIR }}$ with increasing $L_{\text {FIR }}$. Although our data suggest that the [C II] deficit is most probably driven by mechanisms or properties that are intrinsic to the quasar hosts, we do not find evidence for the compactness of the $\mathrm{SF}$ regions being the driver of the $L_{[\mathrm{C} \text { II] }} / L_{\mathrm{FIR}}-L_{\mathrm{FIR}}$ trend.

Our analysis clearly demonstrates the wide variety of host galaxy properties, particularly in terms of SFRs and of possible SMBH fueling mechanisms, among a relatively uniform population of the fastest-growing SMBHs in the early gas-rich universe. It appears that vigorous $\mathrm{SMBH}$ growth is not necessarily accompanied by extreme SF activity (i.e., above what is found in inactive, SF galaxies) and that galaxy-galaxy interactions are not a necessary condition for either of the two processes. This broadly supports a scenario where intense $\mathrm{SMBH}$ and stellar growth in the early universe is driven by secular processes, such as large-scale flows of cold gas, that penetrate into the centers of massive dark matter halos and/or gas or stellar instabilities on smaller scales.

Our results motivate several paths for follow-up studies to address and test the predictions of the different fueling mechanisms. To robustly determine the role of mergers in the systems that lack companions quasars would require the detection of tidal features (e.g., using JWST imaging), or mapping the ISM kinematics at higher resolution and/or to larger scales (i.e., with deeper ALMA observations). Obviously, a critical test of the relevance of mergers to the general population of high-redshift quasars necessitates a significantly larger sample, with observations that cover a large field of view while maintaining a high spatial resolution. This can be achieved by extending our analysis to additional 
$z \sim 5$ and $z \sim 6$ quasars. We have recently guaranteed cycle- 4 ALMA band-7 time to observe 12 additional $z \simeq 4.8$ quasars from the T11 sample, which would allow us to study the host galaxies and close environments of a total of 18 fast-growing, $z \simeq 4.8 \mathrm{SMBHs}$

We thank the anonymous referee for the very constructive comments that helped us to improve our manuscript. We thank K. Schawinski, L. Mayer, R. Teyssier, P. Capelo, M. Dotti, and D. Fiacconi for useful discussions. This paper makes use of the following ALMA data: ADS/JAO.ALMA\#2013.1.01153.S. ALMA is a partnership of ESO (representing its member states), NSF (USA) and NINS (Japan), together with NRC (Canada), NSC and ASIAA (Taiwan), and KASI (Republic of Korea), in cooperation with the Republic of Chile. The Joint ALMA Observatory is operated by ESO, AUI/NRAO, and NAOJ. This work made use of the MATLAB package for astronomy and astrophysics (Ofek 2014). H.N. acknowledges support by the Israel Science Foundation grant 284/13. C.C. gratefully acknowledges support from the Swiss National Science Foundation Professorship grant PP00P2_138979/1. C.C. also acknowledges funding from the European Union's Horizon 2020 research and innovation programme under the Marie Sklodowska-Curie grant agreement No 664931. R.M. acknowledges support by the Science and Technology Facilities Council (STFC) and the ERC Advanced Grant 695671 "QUENCH."

Facility: ALMA (band-7).

Software: CASA.

\section{References}

Adelman-McCarthy, J. K., Agüeros, M. A., Allam, Sahar S., et al. 2008, ApJS, 175,297

Alexander, D. M., \& Hickox, R. C. 2012, NewAR, 56, 93

Aravena, M., Decarli, R., Walter, F., et al. 2016a, ApJ, 833, 68

Aravena, M., Decarli, R., Walter, F., et al. 2016b, ApJ, 833, 71

Bahcall, J. N., Kirhakos, S., Saxe, D. H., \& Schneider, D. P. 1997, ApJ, 479, 642

Baldry, I. K., Driver, S. P., Loveday, J., et al. 2012, MNRAS, 421, 621

Bañados, E., Venemans, B. P., Walter, F., et al. 2013, ApJ, 773, 178

Banerji, M., Carilli, C. L., Jones, G., et al. 2017, MNRAS, 465, 4390

Barnes, J. E. 2002, MNRAS, 333, 481

Becker, G. D., Hewett, P. C., Worseck, G., \& Xavier Prochaska, J. 2013, MNRAS, 430, 2067

Beelen, A., Cox, P., Benford, D. J., et al. 2006, ApJ, 642, 694

Bennert, V. N., Auger, M. W., Treu, T., Woo, J.-H., \& Malkan, M. A. 2011, ApJ, 742, 107

Blecha, L., Cox, T. J., Loeb, A., \& Hernquist, L. 2011, MNRAS, 412, 2154 Bongiorno, A., Maiolino, R., Brusa, M., et al. 2014, MNRAS, 443, 2077 Bouché, N., Dekel, A., Genzel, R., et al. 2010, ApJ, 718, 1001

Bournaud, F., Dekel, A., Teyssier, R., et al. 2011, ApJL, 741, L33 Bournaud, F., Juneau, S., Le Floc'h, E., et al. 2012, ApJ, 757, 81 Bouwens, R. J., Illingworth, G. D., Oesch, P. a., et al. 2015, ApJ, 803, 34 Capak, P. L., Carilli, C. L., Jones, G., et al. 2015, Natur, 522, 455 Capelo, P. R., Volonteri, M., Dotti, M., et al. 2015, MNRAS, 447, 2123 Caplar, N., Lilly, S. J., \& Trakhtenbrot, B. 2015, ApJ, 811, 148 Carilli, C. L., \& Walter, F. 2013, ARA\&A, 51, 105

Carniani, S., Maiolino, R., De Zotti, G., et al. 2015, A\&A, 584, A78 Carniani, S., Marconi, A., Biggs, A., et al. 2013, A\&A, 559, A29

Casey, C. M., Narayanan, D., \& Cooray, A. R. 2014, PhR, 541, 45 Chabrier, G. 2003, PASP, 115,763

Chary, R.-R., \& Elbaz, D. 2001, ApJ, 556, 562

Cicone, C., Maiolino, R., Gallerani, S., et al. 2015, A\&A, 574, A14

Cisternas, M., Jahnke, K., Inskip, K. J., et al. 2011, ApJ, 726, 57

Costa, T., Sijacki, D., Trenti, M., \& Haehnelt, M. G. 2014, MNRAS, 439, 2146 Cox, P., Krips, M., Neri, R., et al. 2011, ApJ, 740, 63

De Breuck, C., Williams, R. J., Swinbank, M., et al. 2014, A\&A, 565, A59

De Rosa, G., Venemans, B. P., Decarli, R., et al. 2014, ApJ, 790, 145

DeBuhr, J., Quataert, E., \& Ma, C.-P. 2011, MNRAS, 412, 1341
Decarli, R., Falomo, R., Treves, A., et al. 2010, MNRAS, 402, 2453

Dekel, A., Birnboim, Y., Engel, G., et al. 2009, Natur, 457, 451

Di Matteo, T., Colberg, J., Springel, V., Hernquist, L., \& Sijacki, D. 2008, ApJ, 676, 33

Di Matteo, T., Khandai, N., DeGraf, C., et al. 2012, ApJL, 745, L29

Di Matteo, T., Springel, V., \& Hernquist, L. 2005, Natur, 433, 604

Díaz-Santos, T., Armus, L., Charmandaris, V., et al. 2013, ApJ, 774, 68

Downes, D., \& Solomon, P. 1998, ApJ, 507, 615

Duncan, K., Conselice, C. J., Mortlock, A., et al. 2014, MNRAS, 444, 2960

Dubois, Y., Pichon, C., Haehnelt, M., et al. 2012, MNRAS, 423, 3616

Dubois, Y., Volonteri, M., Silk, J., et al. 2015, MNRAS, 452, 1502

Dunne, L., Eales, S., Edmunds, M., et al. 2000, MNRAS, 315, 115

Fujimoto, S., Ouchi, M., Ono, Y., et al. 2016, ApJS, 222, 1

Gabor, J. M., Capelo, P. R., Volonteri, M., et al. 2016, A\&A, 592, A62

Gabor, J. M., Impey, C. D., Jahnke, K., et al. 2009, ApJ, 691, 705

Gallerani, S., Neri, R., Maiolino, R., et al. 2012, A\&A, 543, A114

Genel, S., Genzel, R., Bouché, N., Naab, T., \& Sternberg, A. 2009, ApJ, 701, 2002

Gnerucci, A., Marconi, A., Cresci, G., et al. 2011, A\&A, 528, A88

Haring, N., \& Rix, H. 2004, ApJL, 604, L89

Hewett, P. C., \& Wild, V. 2010, MNRAS, 405, 2302

Hodge, J. A., Carilli, C. L., Walter, F., et al. 2012, ApJ, 760, 11

Hopkins, P. F., Croton, D., Bundy, K., et al. 2010, ApJ, 724, 915

Hopkins, P. F., Hernquist, L., Cox, T. J., et al. 2006, ApJS, 163, 1

Husband, K., Bremer, M. N., Stanway, E. R., et al. 2013, MNRAS, 432, 2869

Ilbert, O., McCracken, H. J., Le Fèvre, O., et al. 2013, A\&A, 556, A55

Iono, D., Yun, M. S., Elvis, M., et al. 2006, ApJL, 645, L97

Ivison, R. J., Swinbank, M., Swinyard, B., et al. 2010, A\&A, 518, L35

Karim, A., Swinbank, A. M., Hodge, J. A., et al. 2013, MNRAS, 432, 2

Kim, S., Stiavelli, M., Trenti, M., et al. 2009, ApJ, 695, 809

Knudsen, K. K., Richard, J., Kneib, J.-P., et al. 2016, MNRAS, 462, L6

Kocevski, D. D., Faber, S. M., Mozena, M., et al. 2012, ApJ, 744, 148

Kormendy, J., \& Ho, L. C. 2013, ARA\&A, 51, 511

Koss, M., Mushotzky, R. F., Veilleux, S., et al. 2011, ApJ, 739, 57

Lauer, T. R., Tremaine, S., Richstone, D. O., \& Faber, S. M. 2007, ApJ, 670, 249

Lee, K.-S., Ferguson, H. C., Wiklind, T., et al. 2012, ApJ, 752, 66

Lee, N., Sanders, D. B., Casey, C. M., et al. 2013, ApJ, 778, 131

Leipski, C., Meisenheimer, K., Walter, F., et al. 2014, ApJ, 785, 154

Lilly, S. J., Carollo, C. M., Pipino, A., Renzini, A., \& Peng, Y.-J. 2013, ApJ, 772, 119

Lutz, D., Berta, S., Contursi, A., et al. 2016, A\&A, 591, A136

Lutz, D., Mainieri, V., Rafferty, D. A., et al. 2010, ApJ, 712, 1287

Lyu, J., Rieke, G. H., \& Alberts, S. 2016, ApJ, 816, 85

Magnelli, B., Lutz, D., Saintonge, A., et al. 2014, A\&A, 561, A86

Magnelli, B., Lutz, D., Santini, P., et al. 2012, A\&A, 539, A155

Mainieri, V., Bongiorno, A., Merloni, A., et al. 2011, A\&A, 535, A80

Maiolino, R., Caselli, P., Nagao, T., et al. 2009, A\&A, 500, L1

Maiolino, R., Cox, P., Caselli, P., et al. 2005, A\&A, 440, L51

Maiolino, R., Gallerani, S., Neri, R., et al. 2012, MNRAS, 425, L66

Marconi, A., \& Hunt, L. K. 2003, ApJL, 589, L21

McGreer, I. D., Jiang, L., Fan, X., et al. 2013, ApJ, 768, 105

McLure, R. J., \& Dunlop, J. S. 2004, MNRAS, 352, 1390

Mcmullin, J. P., Waters, B., Schiebel, D., Young, W., \& Golap, K. 2007, in ASP Conf. Ser. 376, Astronomical Data Analysis Software and Systems XVI, ed. R. A. Shaw, F. Hill, \& D. J. Bell (San Francisco, CA: ASP), 127

Mejía-Restrepo, J. E., Trakhtenbrot, B., Lira, P., Netzer, H., \& Capellupo, D. M. 2016, MNRAS, 460, 187

Merloni, A., Bongiorno, A., Bolzonella, M., et al. 2010, ApJ, 708, 137

Mor, R., \& Netzer, H. 2012, MNRAS, 420, 526

Mor, R., Netzer, H., Trakhtenbrot, B., Shemmer, O., \& Lira, P. 2012, ApJL, 749, L25

Muzzin, A., Marchesini, D., Stefanon, M., et al. 2013, ApJ, 777, 18

Neri, R., Downes, D., Cox, P., \& Walter, F. 2014, A\&A, 562, A35

Netzer, H. 2003, ApJL, 583, L5

Netzer, H. 2009, MNRAS, 399, 1907

Netzer, H., Lani, C., Nordon, R., et al. 2016, ApJ, 819, 123

Netzer, H., Lutz, D., Schweitzer, M., et al. 2007, ApJ, 666, 806

Netzer, H., Mor, R., Trakhtenbrot, B., Shemmer, O., \& Lira, P. 2014, ApJ, 791,34

Ofek, E. O. 2014, MATLAB package for astronomy and astrophysics, Astrophysics Source Code Library, ascl:1407.005

Overzier, R. A., Miley, G. K., Bouwens, R. J., et al. 2006, ApJ, 637, 58

Page, M. J., Symeonidis, M., Vieira, J. D., et al. 2012, Natur, 485, 213

Richards, G. T., Kruczek, N. E., Gallagher, S. C., et al. 2011, AJ, 141, 167

Richards, G. T., Strauss, M. A., Fan, X., et al. 2006, AJ, 131, 2766 
Richards, G. T., Vanden Berk, D. E., Reichard, T. A., et al. 2002, AJ, 124,1

Riechers, D. a., Bradford, C. M., Clements, D. L., et al. 2013, Natur, 496, 329

Riechers, D. A., Carilli, C. L., Capak, P. L., et al. 2014, ApJ, 796, 84

Rosario, D. J., Santini, P., Lutz, D., et al. 2012, A\&A, 545, A45

Rosario, D. J., Trakhtenbrot, B., Lutz, D., et al. 2013, A\&A, 560, A72

Sanders, D. B., Soifer, B. T., Elias, J. H., et al. 1988, ApJ, 325, 74

Sani, E., Marconi, A., Hunt, L. K., \& Risaliti, G. 2011, MNRAS, 413, 1479

Sargsyan, L., Samsonyan, A., Lebouteiller, V., et al. 2014, ApJ, 790, 15

Schawinski, K., Simmons, B. D., Urry, C. M., Treister, E., \& Glikman, E. 2012, MNRAS, 425, L61

Schawinski, K., Treister, E., Urry, C. M., et al. 2011, ApJL, 727, L31

Schneider, R., Bianchi, S., Valiante, R., Risaliti, G., \& Salvadori, S. 2015, A\&A, 579, A60

Schweitzer, M., Lutz, D., Sturm, E., et al. 2006, ApJ, 649, 79

Shang, Z., Wills, B. J., Wills, D., \& Brotherton, M. S. 2007, AJ, 134, 294

Shao, L., Lutz, D., Nordon, R., et al. 2010, A\&A, 518, L26

Shen, Y., Brandt, W. N., Denney, K. D., et al. 2016, ApJ, 831, 7

Shen, Y., Richards, G. T., Strauss, M. a., et al. 2011, ApJS, 194, 45

Sijacki, D., Springel, V., \& Haehnelt, M. G. 2009, MNRAS, 400, 100

Sijacki, D., Springel, V., \& Haehnelt, M. G. 2011, MNRAS, 414, 3656

Simpson, C., Mortlock, D. J., Warren, S., et al. 2014, MNRAS, 442, 3454

Speagle, J. S., Steinhardt, C. L., Capak, P. L., \& Silverman, J. D. 2014, ApJS, 214,15

Springel, V., Di Matteo, T., \& Hernquist, L. 2005, MNRAS, 361, 776

Springel, V., \& Hernquist, L. 2005, ApJL, 622, L9

Stacey, G. J., Geis, N., Genzel, R., et al. 1991, ApJ, 373, 423

Stacey, G. J., Hailey-Dunsheath, S., Ferkinhoff, C., et al. 2010, ApJ, 724, 957

Stark, D. P., Ellis, R. S., Bunker, A., et al. 2009, ApJ, 697, 1493

Stefanon, M., Marchesini, D., Muzzin, A., et al. 2015, ApJ, 803, 11
Steinhardt, C. L., Speagle, J. S., Capak, P. L., et al. 2014, ApJL, 791, L25 Swinbank, M., Karim, A., Smail, I., et al. 2012, MNRAS, 427, 1066 Symeonidis, M. 2017, MNRAS, 465, 1401

Symeonidis, M., Giblin, B. M., Page, M. J., et al. 2016, MNRAS, 459, 257

Tacconi, L. J., Genzel, R., Tecza, M., et al. 1999, ApJ, 524, 732

Trakhtenbrot, B., \& Netzer, H. 2010, MNRAS, 406, L35

Trakhtenbrot, B., \& Netzer, H. 2012, MNRAS, 427, 3081

Trakhtenbrot, B., Netzer, H., Lira, P., \& Shemmer, O. 2011, ApJ, 730, 7

Trakhtenbrot, B., Urry, C. M., Civano, F. M., et al. 2015, Sci, 349, 168

Treister, E., Schawinski, K., Urry, C. M., \& Simmons, B. D. 2012, ApJL, 758, L39

Ueda, J., Iono, D., Yun, M. S., et al. 2014, ApJS, 214, 1

Utsumi, Y., Goto, T., Kashikawa, N., et al. 2010, ApJ, 721, 1680

Valiante, R., Schneider, R., Salvadori, S., \& Gallerani, S. 2014, MNRAS, 444,2442

Valtchanov, I., Virdee, J., Ivison, R. J., et al. 2011, MNRAS, 415, 3473

Van Wassenhove, S., Volonteri, M., Mayer, L., et al. 2012, ApJL, 748, L7

Veilleux, S., Kim, D.-C., Rupke, D. S. N., et al. 2009, ApJ, 701, 587

Venemans, B. P., McMahon, R. G., Walter, F., et al. 2012, ApJL, 751, L25

Venemans, B. P., Walter, F., Zschaechner, L., et al. 2016, ApJ, 816, 37

Volonteri, M., Capelo, P. R., Netzer, H., et al. 2015, MNRAS, 449, 1470

Wagg, J., Carilli, C. L., Wilner, D. J., et al. 2010, A\&A, 519, L1

Wagg, J., Wiklind, T., Carilli, C. L., et al. 2012, ApJ, 752, L30

Walter, F., Riechers, D., Cox, P., et al. 2009, Natur, 457, 699

Wang, R., Wagg, J., Carilli, C. L., et al. 2013, ApJ, 773, 24

Williams, R. J., Maiolino, R., Santini, P., et al. 2014, MNRAS, 443, 3780

Willott, C. J., Bergeron, J., \& Omont, A. 2015, ApJ, 801, 123

Willott, C. J., Omont, A., \& Bergeron, J. 2013, ApJ, 770, 13

Willott, C. J., Percival, W. J., McLure, R. J., et al. 2005, ApJ, 626, 657

York, D. G., Adelman, J., Anderson, J. E., Jr., et al. 2000, AJ, 120, 1579 